\title{
Distribution of Benthic Diatom (Phytobenthos) Composition in Küçük Menderes River Basin
}

\section{Küçük Menderes Nehir Havzası'nda Bentik Diyatome (Fitobentoz) Kompozisyonunun Dağılımı}

\author{
Cüneyt Nadir SOLAK ${ }^{1}$, Tolga ÇETIN²${ }^{2}$, Aydın KALEL $\dot{I}^{1}$ \\ ${ }^{1}$ Dumlupinar University, Faculty of Arts and Sciences, Department of Biology, Kütahya \\ cnsolak@gmail.com, aydinkaleli84@gmail.com \\ ${ }^{2}$ T.R. Ministry of Forestry and Water Affairs, Directorate General for Water Management, Ankara \\ tcetin@ormansu.gov.tr \\ Received Date: 09.11.2017, Accepted Date: 26.12.2017
}

\begin{abstract}
Although there are many studies on the diatoms, the most important represantatives of phytobenthos, the distribution of diatom composition is still a new issue in Turkey. This study aims to investigate the distribution of benthic diatom composition in Küçük Menderes River Basin. Samples were collected from epilithon and epipelon substrates of 7 river water bodies and 3 dams (Tahtalı, Beydağı and Alaçatı) on a seasonal basis in 2014. Hydrogen peroxide and hydrochloric acid were used to remove the organic matter from frustules and permanent slides were mounted with Naphrax solution. In total, 94 benthic diatom taxa were identified. Nitzschia palea (Kützing) W.Smith, $N$. inconspicua Grunow, N. umbonata (Ehrenberg) Lange-Bertalot, Craticula accomoda (Hustedt) DG Mann, C. subminuscula (Manguin) C.E.Wetzel \& L.Ector and Navicula veneta Kützing were the most common taxa as an indicator of polluted waters. Achnanthidium eutrophilum (Lange-Bertalot) Lange-Bertalot, $A$. minutissimum var.jackii (Rabenhorst) Lange-Bertalot, Navicula simulata Manguin, N. vandamii Schoeman \& Archibald, Nitzschia archibaldii Lange-Bertalot, $N$. desertorum Hustedt and Sellaphora saprotolerans Lange-Bertalot, Hofmann \& Cantonati were the new records for Turkish diatom flora.

Keywords: Diatom, Küçük Menderes, new records, phytobenthos
\end{abstract}

\section{$\ddot{O} z$}

Fitobentozun en önemli temsilcisi olan diyatomeler ile ilgili pek çok çalışma olmasına rağmen diyatome kompozisyonun dağılımı Türkiye'de halen yeni bir konudur. Bu çalışma ile Küçük Menderes Nehir Havzası'nda bentik diyatome kompozisyonunun dağılımının araştırılması amaçlanmıştır. Örnekler 2014 yılında mevsimsel olarak 7 nehir suyu kütlesinden ve 3 baraj gölünden (Tahtalı, Beydağ 1 ve Alaçatı) epiliton ve epipelon substratlardan alınmıştır. Organik maddenin früstüllerden uzaklaştırılması maksadıyla hidrojen peroksit ve hidroklorik asit kullanılmış ve daimi preparatlar Naphrax solüsyonu ile hazırlanmıştır. Toplamda 94 bentik diyatome taksonu teşhis edilmiştir. Kirli suların indikatörü olan Nitzschia palea (Kützing) W.Smith, $N$. inconspicua Grunow, N. umbonata (Ehrenberg) Lange-Bertalot, Craticula accomoda (Hustedt) DG Mann, C. subminuscula (Manguin) C.E.Wetzel \& L.Ector ve 
Navicula veneta Kützing en yaygın taksonlar olmuştur. Achnanthidium eutrophilum (Lange-Bertalot) Lange-Bertalot, A. minutissimum var. jackii (Rabenhorst) Lange-Bertalot, Navicula simulata Manguin, $N$. vandamii Schoeman \& Archibald, Nitzschia archibaldii Lange-Bertalot, $N$. desertorum Hustedt ve Sellaphora saprotolerans Lange-Bertalot, Hofmann \& Cantonati ise Türkiye diyatome florası için yeni kayitlar olmuştur.

Anahtar sözcükler: Diyatome, Küçük Menderes, yeni kayttlar, fitobentoz

\section{Introduction}

After the implementation of European Union Water Framework Directive (WFD) (2000/60/EC) (Anonymous, 2000) in 2000, ecological assessment came to the forefront in water quality monitoring studies conducted in Europe. European Member States have started to monitor a number of biological quality elements in their water bodies such as phytobenthos, phytoplankton, macrophyte, fish, macroinvertebrate, macroalgae and angiosperms. The harmonization process of the WFD in Turkey has been accelerated since 2011 with the establishment of the Ministry of Forestry and Water Affairs, General Directorate of Water Management (Anonymous, 2011). Biological monitoring studies were carried out in many river basins in Turkey (Anonymous, 2013; Anonymous 2014a; Demir et al. 2017) and are being carried out (Anonymous, 2017) on an ecological basis within the scope of By-law on Monitoring Surface and Ground Waters (Anonymous, 2014b).

The phytobenthos is an obligatory biological quality element to be monitored in rivers and lakes according to the WFD. Phytobenthos contains all phototrophic organisms from microscopic unicellular organisms to macrophytes longer than 2 meters (Anonymous, 2010). Due to the lack of practical methods, EU Member States mostly monitor diatoms in river and lake water bodies as a part of their biological monitoring studies to assess phytobenthos quality element within the scope of the WFD.

Biological monitoring of river basins significantly contributed to the study of flora and fauna in Turkey. Biological data has been obtained from many water bodies that were not studied until today and environmental needs of the taxa were determined (Anonymous, 2016; Toudjani, 2017) with the chemical samples taken at the same time with biological samples. There are number of studies carried out for algal flora in Turkey (Gönülol et al. 1996; Aysel, 2005; Solak et al. 2016) and more than 800 diatom taxa were reported.

Regarding the algal flora studies of Küçük Menderes River Basin, the algal flora of Oğlananası Lake (Gaziemir, İzmir) was identified by Aysel et al. 1998. 40 diatom 
taxa were identified and 10 of them were new records for Turkey. With another study, 86 diatom taxa were identified as a result of sampling on a seasonal basis in 1994 in Barutçu Lake (Selçuk, İzmir). 12 diatom taxa were new records for Turkish flora (Aysel et al. 2002). In addition, the algal flora of Laka River was identified by Aysel et al. 2001. It was found that the river can be used for drinking purposes based on the distribution of the taxa according to algal divisions. This study aims to investigate the distribution of benthic diatom composition and to prepare the taxa catalogue according to the diatom taxa obtained from the water quality monitoring study in the Küçük Menderes River Basin, and to benefit from the results for future monitoring studies carried out in the other river basins of Turkey.

\section{Method}

\section{Study Site}

Küçük Menderes River Basin is placed in the west of Turkey between Büyük Menderes and Gediz River Basins and its waters flow into the Aegean Sea. River basin is located between $38^{\circ} 41^{\prime} 05^{\prime \prime}$ and $37^{\circ} 24^{\prime} 08^{\prime \prime}$ northern latitudes with $28^{\circ} 24^{\prime} 36^{\prime \prime}$ and $26^{\circ} 11^{\prime} 48^{\prime \prime}$ eastern longitudes. Surface area of this basin is 702.931 ha and it corresponds to $0.9 \%$ of the surface area of Turkey (Anonymous, 2014c).

\section{Sampling}

Samples were collected from 7 river water bodies and 3 dams (Table 1) in Küçük Menderes River Basin (Figure 1) in May, July and September in 2014. Epilithic samples were taken by brushing the submerged stones, and epipelic samples were taken by using a pipette aspirator from the sediment.

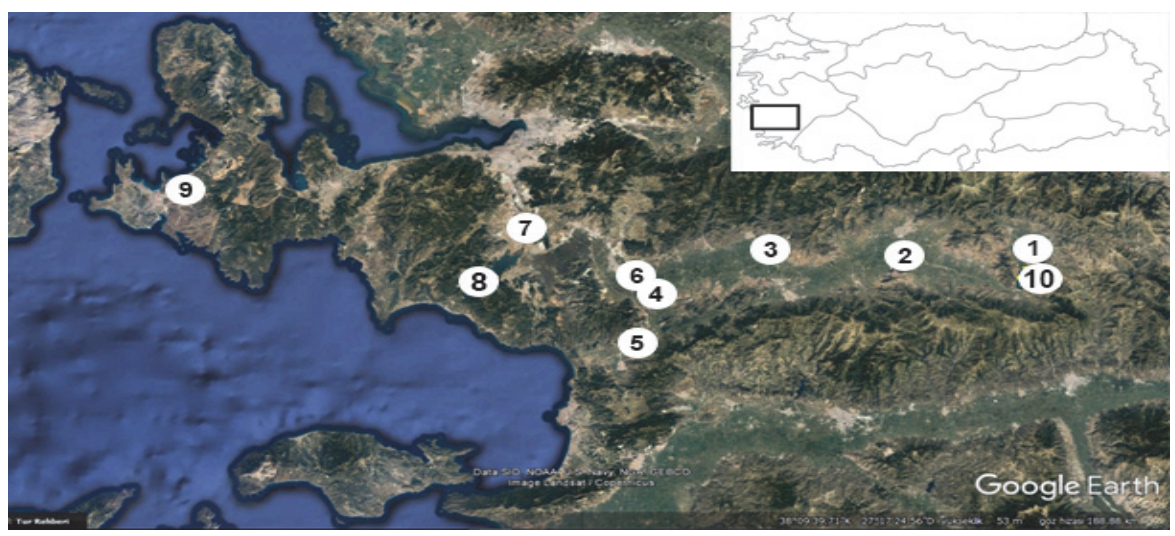

Figure 1. Distribution of sampling points in Küçük Menderes River Basin 
Table 1

The Coordinates of The Sampling Points of The Water Bodies in Küçük Menderes River Basin

\begin{tabular}{cccc}
\hline Stations & Water Bodies & \multicolumn{2}{c}{ Coordinates } \\
\hline K1 & Keleş Creek & $38^{\circ} 09^{\prime} 55.27^{\prime \prime} \mathrm{N}$ & $28^{\circ} 13^{\prime} 10.55^{\prime \prime} \mathrm{E}$ \\
K2 & Küçük Menderes River & $38^{\circ} 09^{\prime} 56.39^{\prime \prime} \mathrm{N}$ & $27^{\circ} 57^{\prime} 51.15^{\prime \prime} \mathrm{E}$ \\
K3 & Ilıca Creek & $38^{\circ} 09^{\prime} 11.05^{\prime \prime} \mathrm{N}$ & $27^{\circ} 40^{\prime} 56.53^{\prime \prime} \mathrm{E}$ \\
K4 & Fetrek Creek & $38^{\circ} 04^{\prime} 23.92^{\prime \prime} \mathrm{N}$ & $27^{\circ} 24^{\prime} 52.89^{\prime \prime} \mathrm{E}$ \\
K5 & Küçük Menderes River & $37^{\circ} 58^{\prime} 41.05^{\prime \prime} \mathrm{N}$ & $27^{\circ} 22^{\prime} 44.02^{\prime \prime} \mathrm{E}$ \\
K6 & Küçük Menderes River & $38^{\circ} 05^{\prime} 46.76^{\prime \prime} \mathrm{N}$ & $27^{\circ} 23^{\prime} 45.49^{\prime \prime} \mathrm{E}$ \\
K7 & Sang1 Creek & $38^{\circ} 11^{\prime} 47.24^{\prime \prime} \mathrm{N}$ & $27^{\circ} 09^{\prime} 59.25^{\prime \prime} \mathrm{E}$ \\
K8 & Tahtalı Dam & $38^{\circ} 05^{\prime} 26.38^{\prime \prime} \mathrm{N}$ & $27^{\circ} 02^{\prime} 58.32^{\prime \prime} \mathrm{E}$ \\
K9 & Alaçatı Dam & $38^{\circ} 17^{\prime} 03.24^{\prime \prime} \mathrm{N}$ & $26^{\circ} 24^{\prime} 28.46^{\prime \prime} \mathrm{E}$ \\
K10 & Beydağ Dam & $38^{\circ} 06^{\prime} 32.42^{\prime \prime} \mathrm{N}$ & $28^{\circ} 13^{\prime} 11.18^{\prime \prime} \mathrm{E}$ \\
\hline
\end{tabular}

\section{Sample Processing, Observation and Identification}

Samples were boiled with $\mathrm{H}_{2} \mathrm{O}_{2}$ and $\mathrm{HCl}$ to remove the organic matter from frustules. After washing three times of diatoms with distilled water, the material was air-dried on cover glasses and mounted with Naphrax solution. Diatoms were observed with a Nikon Ci Light Microscope (LM) in Dumlupınar University, Turkey.

The diatoms were identified according to Hofmann et al. (2011); Krammer and Lange-Bertalot (1998, 1991); Lange-Bertalot (2001); Levkov et al. (2013); Wojtal (2009) and Wojtal and Kwandrans (2006). Reported dimensions (length, width, number of striae $/ 10 \mu \mathrm{m}$ ) of each taxa were based on the measurements performed in this study.

\section{Diatom Distribution}

Description of the distribution of Turkish diatom flora was made according to Gönülol (2017). The taxa reported from Turkey by Gönülol (2017) which cover 10\% of the literature are categorized as "common" diatoms, the ones cover less than $10 \%$ are noted as "rare" diatoms. For each station, about 400 valves were counted and then relative abundances were calculated. If the taxon exists in 1 or 2 sampling station(s), it was named as "rare" and if it exists in more than 2 stations, it was named as "common" in this study. 


\section{Results}

A total of 94 taxa were identified and 7 of those were recorded as new taxa for Turkish diatom flora. The taxa identified in Küçük Menderes River Basin and indicated by Gönülol (2017) for Turkey as a whole are listed in Table 2 and brief notes, dimensions and distribution of each diatom are provided.

Table 2

The Distribution of The Diatom Taxa in Turkey (Gönülol, 2017) and in This Study

\begin{tabular}{|c|c|c|c|}
\hline & \multirow{2}{*}{$\begin{array}{l}\text { Status in Turkey } \\
\text { (Gönülol, 2017) }\end{array}$} & \multicolumn{2}{|c|}{ In This Study } \\
\hline & & Status & Station(s) \\
\hline $\begin{array}{l}\text { Achnanthidium eutrophilum } \\
\text { (Lange-Bertalot) Lange-Bertalot }\end{array}$ & NR & $\mathrm{R}$ & K10 \\
\hline $\begin{array}{l}\text { Achnanthidium exiguum } \\
\text { (Grunow) Czarnecki }\end{array}$ & $\mathrm{R}$ & $\mathrm{R}$ & K5 \\
\hline $\begin{array}{l}\text { Achnanthidium minutissimum } \\
\text { (Kützing) Czarnecki }\end{array}$ & $\mathrm{C}$ & $\mathrm{C}$ & K7, K8, K9 \\
\hline $\begin{array}{l}\text { Achnanthidium inutissimum var. jackii } \\
\text { (Rabenhorst) Lange-Bertalot }\end{array}$ & NR & $\mathrm{R}$ & K8 \\
\hline $\begin{array}{l}\text { Adlafia minuscula } \\
\text { (Grunow) Lange-Bertalot }\end{array}$ & $\mathrm{C}$ & $\mathrm{R}$ & K1 \\
\hline $\begin{array}{l}\text { Amphora pediculus } \\
\text { (Kützing) Grunow ex A.Schmidt }\end{array}$ & $\mathrm{C}$ & $\mathrm{R}$ & K1, K7 \\
\hline $\begin{array}{l}\text { Aulacoseira ambigua } \\
\text { (Grunow) Simonsen }\end{array}$ & $\mathrm{R}$ & $\mathrm{R}$ & K10 \\
\hline $\begin{array}{l}\text { Cocconeis pediculus } \\
\text { Ehrenberg }\end{array}$ & $\mathrm{C}$ & $\mathrm{C}$ & K1, K7, K9 \\
\hline $\begin{array}{l}\text { Craticula accomoda } \\
\text { (Hustedt) DG Mann }\end{array}$ & $\mathrm{C}$ & $\mathrm{C}$ & $\mathrm{K} 1, \mathrm{~K} 3, \mathrm{~K} 4, \mathrm{~K} 5, \mathrm{~K} 8, \mathrm{~K} 10$ \\
\hline $\begin{array}{l}\text { Craticula cuspidate } \\
\text { (Kützing) DG Mann }\end{array}$ & $\mathrm{C}$ & $\mathrm{R}$ & K7 \\
\hline $\begin{array}{l}\text { Craticula melostiformis } \\
\text { (Hustedt) Mayama }\end{array}$ & $\mathrm{R}$ & $\mathrm{C}$ & K2, K3, K9 \\
\hline $\begin{array}{l}\text { Craticula subminuscula } \\
\text { (Manguin) C.E.Wetzel \& L.Ector }\end{array}$ & $\mathrm{C}$ & $\mathrm{C}$ & $\mathrm{K} 1, \mathrm{~K} 2, \mathrm{~K} 3, \mathrm{~K} 5, \mathrm{~K} 9, \mathrm{~K} 10$ \\
\hline $\begin{array}{l}\text { Cyclostephanos dubius } \\
\text { (Hustedt) Round }\end{array}$ & $\mathrm{R}$ & $\mathrm{C}$ & K6, K9, K10 \\
\hline $\begin{array}{l}\text { Cyclostephanos invisitatus } \\
\text { (Hohn \& Hellermann) Theriot, } \\
\text { Stoermer \& Håkasson }\end{array}$ & $\mathrm{R}$ & $\mathrm{R}$ & K10 \\
\hline $\begin{array}{l}\text { Cyclotella atomus } \\
\text { Hustedt }\end{array}$ & $\mathrm{R}$ & $\mathrm{R}$ & $\mathrm{K} 2$ \\
\hline
\end{tabular}


Table 2 (continue)

The Distribution of The Diatom Taxa in Turkey (Gönülol, 2017) and in This Study

\begin{tabular}{|c|c|c|c|}
\hline & \multirow{2}{*}{$\begin{array}{l}\text { Status in Turkey } \\
\text { (Gönülol, 2017) }\end{array}$} & \multicolumn{2}{|c|}{ In This Study } \\
\hline & & Status & Station(s) \\
\hline $\begin{array}{l}\text { Cyclotella meneghiniana } \\
\text { Kützing }\end{array}$ & $\mathrm{C}$ & $\mathrm{C}$ & K3, K5, K7 \\
\hline $\begin{array}{l}\text { Cymbella cymbiformis } \\
\text { C. Agardh }\end{array}$ & $\mathrm{C}$ & $\mathrm{R}$ & K8 \\
\hline $\begin{array}{l}\text { Cymbella excise } \\
\text { Kützing }\end{array}$ & $\mathrm{R}$ & $\mathrm{R}$ & K8, K9 \\
\hline $\begin{array}{l}\text { Cymbella lange-bertalotii } \\
\text { Krammer }\end{array}$ & $\mathrm{R}$ & $\mathrm{R}$ & K8 \\
\hline $\begin{array}{l}\text { Cymbella parva } \\
\text { (W. Smith) Cleve }\end{array}$ & $\mathrm{C}$ & $\mathrm{R}$ & K7 \\
\hline $\begin{array}{l}\text { Cymbopleura amphicephala } \\
\text { Näegeli }\end{array}$ & $\mathrm{C}$ & $\mathrm{R}$ & K7 \\
\hline $\begin{array}{l}\text { Diatoma moniliformis } \\
\text { (Kützing) D.M.Williams }\end{array}$ & $\mathrm{C}$ & $\mathrm{R}$ & K4, K5 \\
\hline $\begin{array}{l}\text { Encyonema caespitosum } \\
\text { Kützing }\end{array}$ & $\mathrm{C}$ & $\mathrm{R}$ & K8 \\
\hline $\begin{array}{l}\text { Encyonema ventricosum } \\
\text { (C.Agardh) Grunow }\end{array}$ & $\mathrm{C}$ & $\mathrm{C}$ & K1, K6, K7 \\
\hline $\begin{array}{l}\text { Encyonopsis microcephala } \\
\text { (Grunow) Krammer }\end{array}$ & $\mathrm{C}$ & $\mathrm{R}$ & K6, K7 \\
\hline $\begin{array}{l}\text { Encyonopsis minuta } \\
\text { Krammer \& Reichardt }\end{array}$ & $\mathrm{R}$ & $\mathrm{C}$ & $\mathrm{K} 6, \mathrm{~K} 7, \mathrm{~K} 8$ \\
\hline $\begin{array}{l}\text { Encyonopsis subminuta } \\
\text { Krammer \& Reichardt }\end{array}$ & $\mathrm{R}$ & $\mathrm{C}$ & $\mathrm{K} 6, \mathrm{~K} 7, \mathrm{~K} 8, \mathrm{~K} 10$ \\
\hline $\begin{array}{l}\text { Fallacia pygmeae } \\
\text { (Kützing) Stikle et Mann }\end{array}$ & $\mathrm{C}$ & $\mathrm{R}$ & K10 \\
\hline $\begin{array}{l}\text { Fragilaria capucina var. vaucheria } \\
\text { (Kützing) Lange-Bertalot }\end{array}$ & $\mathrm{C}$ & $\mathrm{R}$ & $\mathrm{K} 8, \mathrm{~K} 10$ \\
\hline $\begin{array}{l}\text { Fragilaria mesolepta } \\
\text { Rabenhorst }\end{array}$ & $\mathrm{C}$ & $\mathrm{R}$ & K8 \\
\hline $\begin{array}{l}\text { Fragilaria perminuta } \\
\text { (Grunow) Lange-Bertalot }\end{array}$ & $\mathrm{R}$ & $\mathrm{R}$ & K8, K9 \\
\hline $\begin{array}{l}\text { Geissleria decussis } \\
\text { (Østrup) Lange-Bertalot \& Metzeltin }\end{array}$ & $\mathrm{C}$ & $\mathrm{R}$ & K1 \\
\hline $\begin{array}{l}\text { Gomphonema olivaceum } \\
\text { (Hornemann) Brébisson }\end{array}$ & $\mathrm{C}$ & $\mathrm{R}$ & K7, K8 \\
\hline $\begin{array}{l}\text { Gomphonema parvulum } \\
\text { (Kützing) Kützing }\end{array}$ & $\mathrm{C}$ & $\mathrm{C}$ & $\mathrm{K} 1, \mathrm{~K} 4, \mathrm{~K} 5$ \\
\hline $\begin{array}{l}\text { Gomphonema pumilum } \\
\text { (Grunow) E.Reichardt \& } \\
\text { Lange-Bertalot }\end{array}$ & $\mathrm{R}$ & $\mathrm{C}$ & K1, K6, K7 \\
\hline
\end{tabular}


Table 2 (continue)

The Distribution of The Diatom Taxa in Turkey (Gönülol, 2017) and in This Study

\begin{tabular}{|c|c|c|c|}
\hline & \multirow{2}{*}{$\begin{array}{l}\text { Status in Turkey } \\
\text { (Gönülol, 2017) }\end{array}$} & \multicolumn{2}{|c|}{ In This Study } \\
\hline & & Status & Station(s) \\
\hline $\begin{array}{l}\text { Halamphora montana } \\
\text { (Krasske) Levkov }\end{array}$ & $\mathrm{R}$ & $\mathrm{C}$ & K3, K5, K10 \\
\hline $\begin{array}{l}\text { Halamphora veneta } \\
\text { (Kützing) Levkov }\end{array}$ & $\mathrm{C}$ & C & K1, K2, K5, K9 \\
\hline $\begin{array}{l}\text { Hantzschia amphioxys } \\
\text { (Ehrenberg) Grunow }\end{array}$ & $\mathrm{C}$ & $\mathrm{R}$ & K1 \\
\hline $\begin{array}{l}\text { Hippodonta capitate } \\
\text { (Ehrenberg) Lange-Bertalot, } \\
\text { Metzeltin et Witkowski }\end{array}$ & $\mathrm{C}$ & $\mathrm{R}$ & K10 \\
\hline $\begin{array}{l}\text { Luticola goeppertiana } \\
\text { (Bleisch) D.G.Mann }\end{array}$ & $\mathrm{R}$ & $\mathrm{R}$ & K5 \\
\hline $\begin{array}{l}\text { Luticola ventricosa } \\
\text { (Kützing) D.G.Mann }\end{array}$ & $\mathrm{R}$ & $\mathrm{R}$ & K5 \\
\hline $\begin{array}{l}\text { Mayameae permitis } \\
\text { (Hustedt) K.Bruder \& L.K. Medlin }\end{array}$ & $\mathrm{R}$ & $\mathrm{C}$ & $\mathrm{K} 2, \mathrm{~K} 3, \mathrm{~K} 4, \mathrm{~K} 10$ \\
\hline $\begin{array}{l}\text { Melosira varians } \\
\text { Agardh }\end{array}$ & $\mathrm{C}$ & $\mathrm{R}$ & K10 \\
\hline $\begin{array}{l}\text { Navicula antonii } \\
\text { Lange-Bertalot }\end{array}$ & $\mathrm{R}$ & $\mathrm{R}$ & $\mathrm{K} 1, \mathrm{~K} 7$ \\
\hline $\begin{array}{l}\text { Navicula capitatoradiata } \\
\text { Germain }\end{array}$ & $\mathrm{C}$ & $\mathrm{C}$ & K4, K7, K8, K9 \\
\hline $\begin{array}{l}\text { Navicula cryptocephala } \\
\text { Kützing }\end{array}$ & $\mathrm{C}$ & $\mathrm{C}$ & $\mathrm{K} 4, \mathrm{~K} 7, \mathrm{~K} 8, \mathrm{~K} 9$ \\
\hline $\begin{array}{l}\text { Navicula cryptotenella } \\
\text { Lange-Bertalot }\end{array}$ & $\mathrm{C}$ & $\mathrm{R}$ & K9 \\
\hline $\begin{array}{l}\text { Navicula cryptotenelloides } \\
\text { Lange-Bertalot }\end{array}$ & $\mathrm{R}$ & $\mathrm{R}$ & K8 \\
\hline $\begin{array}{l}\text { Navicula erifuga } \\
\text { Lange-Bertalot }\end{array}$ & $\mathrm{R}$ & $\mathrm{R}$ & K10 \\
\hline $\begin{array}{l}\text { Navicula gregaria } \\
\text { Donkin }\end{array}$ & $\mathrm{C}$ & $\mathrm{C}$ & $\mathrm{K} 1, \mathrm{~K} 2, \mathrm{~K} 3, \mathrm{~K} 10$ \\
\hline $\begin{array}{l}\text { Navicula notha } \\
\text { Wallace }\end{array}$ & $\mathrm{R}$ & $\mathrm{R}$ & K10 \\
\hline $\begin{array}{l}\text { Navicula radiosa } \\
\text { Kützing }\end{array}$ & $\mathrm{C}$ & $\mathrm{R}$ & K8 \\
\hline $\begin{array}{l}\text { Navicula reichardtiana } \\
\text { Lange-Bertalot }\end{array}$ & $\mathrm{R}$ & $\mathrm{R}$ & K1 \\
\hline $\begin{array}{l}\text { Navicula simulate } \\
\text { Manguin }\end{array}$ & NR & $\mathrm{R}$ & K2, K9 \\
\hline $\begin{array}{l}\text { Navicula trivialis } \\
\text { Lange-Bertalot }\end{array}$ & $\mathrm{C}$ & $\mathrm{R}$ & K9 \\
\hline
\end{tabular}


Table 2 (continue)

The Distribution of The Diatom Taxa in Turkey (Gönülol, 2017) and in This Study

\begin{tabular}{|c|c|c|c|}
\hline & \multirow{2}{*}{$\begin{array}{l}\text { Status in Turkey } \\
\text { (Gönülol, 2017) }\end{array}$} & \multicolumn{2}{|c|}{ In This Study } \\
\hline & & Status & Station(s) \\
\hline $\begin{array}{l}\text { Navicula vandamii } \\
\text { Schoeman \& Archibald }\end{array}$ & NR & $\mathrm{R}$ & K2, K9 \\
\hline $\begin{array}{l}\text { Navicula veneta } \\
\text { Kützing }\end{array}$ & $\mathrm{C}$ & $\mathrm{C}$ & K1, K2, K4, K5, K6, K10 \\
\hline $\begin{array}{l}\text { Navicula viridula var. germainii } \\
\text { (Wallace) Lange-Bertalot }\end{array}$ & $\mathrm{R}$ & $\mathrm{R}$ & $\mathrm{K} 2, \mathrm{~K} 10$ \\
\hline $\begin{array}{l}\text { Navicula viridula var. rostellata } \\
\text { (Kützing) Cleve }\end{array}$ & $\mathrm{C}$ & $\mathrm{R}$ & $\mathrm{K} 10$ \\
\hline $\begin{array}{l}\text { Nitzschia amphibia } \\
\text { Grunow }\end{array}$ & $\mathrm{C}$ & $\mathrm{R}$ & $\mathrm{K} 4, \mathrm{~K} 5$ \\
\hline $\begin{array}{l}\text { Nitzschia archibaldii } \\
\text { Lange-Bertalot }\end{array}$ & NR & $\mathrm{C}$ & $\mathrm{K} 2, \mathrm{~K} 3, \mathrm{~K} 10$ \\
\hline $\begin{array}{l}\text { Nitzschia capitellata } \\
\text { Hustedt in A. Schmidt }\end{array}$ & $\mathrm{C}$ & $\mathrm{C}$ & $\mathrm{K} 2, \mathrm{~K} 3, \mathrm{~K} 4, \mathrm{~K} 10$ \\
\hline $\begin{array}{l}\text { Nitzschia desertorum } \\
\text { Hustedt }\end{array}$ & NR & $\mathrm{R}$ & $\mathrm{K} 2$ \\
\hline $\begin{array}{l}\text { Nitzschia dissipata } \\
\text { (Kützing) Grunow }\end{array}$ & $\mathrm{C}$ & $\mathrm{R}$ & K9 \\
\hline $\begin{array}{l}\text { Nitzschia dissipata var. media } \\
\text { (Hantzsch) Grunow }\end{array}$ & $\mathrm{C}$ & $\mathrm{R}$ & K9 \\
\hline $\begin{array}{l}\text { Nitzschia filiformis } \\
\text { (W.Smith) Van Heurck }\end{array}$ & $\mathrm{C}$ & $\mathrm{R}$ & K9 \\
\hline $\begin{array}{l}\text { Nitzschia fonticola } \\
\text { (Grunow) Grunow in Van Heurck }\end{array}$ & $\mathrm{C}$ & $\mathrm{R}$ & $\mathrm{K} 1, \mathrm{~K} 3$ \\
\hline $\begin{array}{l}\text { Nitzschia frustulum } \\
\text { (Kützing) Grunow }\end{array}$ & $\mathrm{C}$ & $\mathrm{R}$ & K9, K10 \\
\hline $\begin{array}{l}\text { Nitzschia inconspicua } \\
\text { Grunow }\end{array}$ & $\mathrm{C}$ & $\mathrm{C}$ & $\mathrm{K} 1, \mathrm{~K} 4, \mathrm{~K} 8, \mathrm{~K} 9, \mathrm{~K} 10$ \\
\hline $\begin{array}{l}\text { Nitzschia linearis } \\
\text { (C.Agardh) W. Smith }\end{array}$ & $\mathrm{C}$ & $\mathrm{R}$ & $\mathrm{K} 1$ \\
\hline $\begin{array}{l}\text { Nitzschia palea } \\
\text { (Kützing) W.Smith }\end{array}$ & $\mathrm{C}$ & $\mathrm{C}$ & $\begin{array}{c}\mathrm{K} 1, \mathrm{~K} 2, \mathrm{~K} 3, \mathrm{~K} 4, \mathrm{~K} 5, \mathrm{~K} 6, \mathrm{~K} 7, \\
\mathrm{~K} 10\end{array}$ \\
\hline $\begin{array}{l}\text { Nitzschia sociabilis } \\
\text { Hustedt }\end{array}$ & $\mathrm{R}$ & $\mathrm{R}$ & K9 \\
\hline $\begin{array}{l}\text { Nitzschia umbonata } \\
\text { (Ehrenberg) Lange-Bertalot }\end{array}$ & $\mathrm{C}$ & $\mathrm{C}$ & $\mathrm{K} 1, \mathrm{~K} 2, \mathrm{~K} 4, \mathrm{~K} 5$, K6 \\
\hline $\begin{array}{l}\text { Pantocsekiella ocellata } \\
\text { (Pantocsek) K.T.Kiss \& Ács } \\
\text { Planothidium lanceolatum }\end{array}$ & $\mathrm{C}$ & $\mathrm{R}$ & K8 \\
\hline $\begin{array}{l}\text { Planothidium lanceolatum } \\
\text { (Brébisson ex Kützing) } \\
\text { Lange-Bertalot }\end{array}$ & $\mathrm{C}$ & $\mathrm{R}$ & $\mathrm{K} 1, \mathrm{~K} 8$ \\
\hline
\end{tabular}


Table 2 (continue)

The Distribution of The Diatom Taxa in Turkey (Gönülol, 2017) and in This Study

\begin{tabular}{|c|c|c|c|}
\hline & \multirow{2}{*}{$\begin{array}{l}\text { Status in Turkey } \\
\text { (Gönülol, 2017) }\end{array}$} & \multicolumn{2}{|c|}{ In This Study } \\
\hline & & Status & Station(s) \\
\hline $\begin{array}{l}\text { Pseudofallacia monoculata } \\
\text { (Hustedt) D.G.Mann }\end{array}$ & $\mathrm{R}$ & $\mathrm{R}$ & $\mathrm{K} 3, \mathrm{~K} 10$ \\
\hline $\begin{array}{l}\text { Pseudostaurosira brevistriata } \\
\text { (Grunow) D.M.Williams \& Round }\end{array}$ & $\mathrm{C}$ & $\mathrm{R}$ & K8 \\
\hline $\begin{array}{l}\text { Reimeria sinuate } \\
\text { (Gregory) Kociolek et Stoermer }\end{array}$ & $\mathrm{C}$ & $\mathrm{C}$ & $\mathrm{K} 1, \mathrm{~K} 2, \mathrm{~K} 7$ \\
\hline $\begin{array}{l}\text { Sellaphora pupula } \\
\text { (Kützing) Mereschkovsky }\end{array}$ & $\mathrm{C}$ & $\mathrm{C}$ & $\mathrm{K} 1, \mathrm{~K} 2, \mathrm{~K} 3, \mathrm{~K} 10$ \\
\hline $\begin{array}{l}\text { Sellaphora radiosa } \\
\text { (Hustedt) H.Kobayasi } \\
\text { Sellaphora saprotolerans }\end{array}$ & $\mathrm{R}$ & $\mathrm{R}$ & K5 \\
\hline $\begin{array}{l}\text { Lange-Bertalot, } \\
\text { Hofmann \& Cantonati }\end{array}$ & NR & $\mathrm{C}$ & $\mathrm{K} 1, \mathrm{~K} 2, \mathrm{~K} 10$ \\
\hline $\begin{array}{l}\text { Sellaphora saugerresii } \\
\text { (Desmazières) C.E.Wetzel \& } \\
\text { D.G.Mann }\end{array}$ & $\mathrm{C}$ & $\mathrm{C}$ & $\mathrm{K} 1, \mathrm{~K} 2, \mathrm{~K} 3, \mathrm{~K} 4$ \\
\hline $\begin{array}{l}\text { Sellaphora seminulum } \\
\text { (Grunow) D.G.Mann }\end{array}$ & $\mathrm{R}$ & $\mathrm{R}$ & K4, K5 \\
\hline $\begin{array}{l}\text { Stephanodiscus hantzschii } \\
\text { Grunow }\end{array}$ & $\mathrm{C}$ & $\mathrm{R}$ & K10 \\
\hline $\begin{array}{l}\text { Surirella brebissonii } \\
\text { Krammer et Lange-Bertalot }\end{array}$ & $\mathrm{C}$ & $\mathrm{C}$ & $\mathrm{K} 1, \mathrm{~K} 2, \mathrm{~K} 5$ \\
\hline $\begin{array}{l}\text { Surirella minuta } \\
\text { Brébisson }\end{array}$ & $\mathrm{C}$ & $\mathrm{R}$ & $\mathrm{K} 2$ \\
\hline $\begin{array}{l}\text { Surirella robusta } \\
\text { Ehrenberg }\end{array}$ & $\mathrm{C}$ & $\mathrm{C}$ & K1, K2, K10 \\
\hline $\begin{array}{l}\text { Tryblionella apiculate } \\
\text { Gregory }\end{array}$ & $\mathrm{C}$ & $\mathrm{R}$ & $\mathrm{K} 2$ \\
\hline $\begin{array}{l}\text { Tryblionella calida } \\
\text { (Grunow) D.G.Mann }\end{array}$ & $\mathrm{R}$ & $\mathrm{R}$ & K2 \\
\hline $\begin{array}{l}\text { Ulnaria acus } \\
\text { (Nitzsch) P.Compère }\end{array}$ & $\mathrm{C}$ & $\mathrm{R}$ & K8 \\
\hline $\begin{array}{l}\text { Ulnaria biceps } \\
\text { (Kützing) P.Compère }\end{array}$ & $\mathrm{C}$ & $\mathrm{C}$ & $\mathrm{K} 5, \mathrm{~K} 7, \mathrm{~K} 8$ \\
\hline $\begin{array}{l}\text { Ulnaria capitate } \\
\text { (Ehrenberg) Compère }\end{array}$ & $\mathrm{C}$ & $\mathrm{R}$ & K8 \\
\hline $\begin{array}{l}\text { Ulnaria delicatissima } \\
\text { (W.Smith) M.Aboal \& P.C.Silva }\end{array}$ & $\mathrm{C}$ & $\mathrm{R}$ & K6, K8 \\
\hline $\begin{array}{l}\text { Ulnaria ulna } \\
\text { (Nitzsch) P.Compère }\end{array}$ & $\mathrm{C}$ & $\mathrm{R}$ & K1 \\
\hline
\end{tabular}

Note. NR: New Record, C: Common, R: Rare 
Achnanthidium eutrophilum (Lange-Bertalot) Lange-Bertalot (Figure 2)

Basionym: Achnanthes eutrophila Lange-Bertalot

Ref: Hofmann et al. 2011 p.80-pl.23: 30-35

Dimensions: Valve 9.4-10.2 $\mu \mathrm{m}$ length, 3.2-3.7 $\mu \mathrm{m}$ width and 25-26 striae in $10 \mu \mathrm{m}$

Distribution in Küçük Menderes River Basin: K10

Achnanthidium exiguum (Grunow) Czarnecki (Figure 3)

Basionym: Achnanthes exigua Grunow

Ref: Hofmann et al. 2011 p.70-pl.22: 14-20

Dimensions: Valve 11.8-12.9 $\mu \mathrm{m}$ length, 4.2-4.8 $\mu \mathrm{m}$ width and 25-26 striae in $10 \mu \mathrm{m}$

Distribution in Küçük Menderes River Basin: K5

Achnanthidium minutissimum (Kützing) Czarnecki (Figure 4)

Basionym: Achnanthes minutissima Kützing

Ref: Hofmann et al. 2011 p.83-pl.23: 15-21

Dimensions: Valve 9.5-11.2 $\mu \mathrm{m}$ length and 2.5-3.1 $\mu \mathrm{m}$ width

Distribution in Küçük Menderes River Basin: K7, K8, K9

Achnanthidium minutissimum var. jackii (Rabenhorst) Lange-Bertalot (Figure 5) Basionym: Achnanthidium jackii Rabenhorst

Ref: Hofmann et al. 2011 p.84-pl.23: 22-29

Dimensions: Valve 9.5-11.2 $\mu \mathrm{m}$ length and 2.5-3.1 $\mu \mathrm{m}$ width

Distribution in Küçük Menderes River Basin: K8

Adlafia minuscula (Grunow) Lange-Bertalot (Figure 6)

Basionym: Navicula minuscula Grunow

Ref: Hofmann et al. 2011 p.91-pl.42: 76-80

Dimensions: Valve 10.2-11.4 $\mu \mathrm{m}$ length and 3.1-3.7 $\mu \mathrm{m}$ width

Distribution in Küçük Menderes River Basin: K1

\section{Amphora pediculus (Kützing) Grunow ex A.Schmidt (Figure 7)}

Basionym: Cymbella pediculus Kützing

Ref: Hofmann et al. 2011 p.98-pl.91: 29-33

Dimensions: Valve 8.3-12.4 $\mu \mathrm{m}$ length and 2.6-3.1 $\mu \mathrm{m}$ width

Distribution in Küçük Menderes River Basin: K1, K7

Aulacoseira ambigua (Grunow) Simonsen (Figure 8)

Basionym: Melosira crenulata var. ambigua Grunow

Ref: Krammer \& Lange-Bertalot 1991 p. 25-p1.21: 1-16

Dimensions: Valve 4.7-5.5 $\mu \mathrm{m}$ length, 8.5-14.3 $\mu \mathrm{m}$ width, $15-18$ interstriae in $10 \mu \mathrm{m}$

Distribution in Küçük Menderes River Basin: K10 
Cocconeis pediculus Ehrenberg (Figure 9)

Ref: Hofmann et al. 2011 p.132-pl.19: 17-19

Dimensions: Valve 23.4-31.4 $\mu \mathrm{m}$ length, 21.8-24.2 $\mu \mathrm{m}$ width, 18-21 striae in $10 \mu \mathrm{m}$

Distribution in Küçük Menderes River Basin: K1, K7, K9

Craticula accomoda (Hustedt) DG Mann (Figure 10)

Basionym: Navicula accomoda Hustedt

Ref: Lange-Bertalot 2001 p.108-pl.93: 1-6

Dimensions: Valve 18.3-23.2 $\mu \mathrm{m}$ length, 5.8-7.1 $\mu \mathrm{m}$ width and 23-25 striae in $10 \mu \mathrm{m}$

Distribution in Küçük Menderes River Basin: K1, K3, K4, K5, K8, K10

Craticula cuspidata (Kützing) DG Mann (Figure 11)

Basionym: Frustulia cuspidata Kützing

Ref: Lange-Bertalot 2001 p.111-pl.82: 1-3; 82: 1, 2

Dimensions: Valve 73.8-85.3 $\mu \mathrm{m}$ length, 5.8-7.1 $\mu \mathrm{m}$ width and 23-25 striae in $10 \mu \mathrm{m}$

Distribution in Küçük Menderes River Basin: K7

Craticula molestiformis (Hustedt) Mayama (Figure 12)

Basionym: Navicula minusculoides Hustedt

Ref: Lange-Bertalot 2001 p.115-pl.93: 7-15

Dimensions: Valve 14.5-16.7 $\mu \mathrm{m}$ length and 3.1-3.7 $\mu \mathrm{m}$ width

Distribution in Küçük Menderes River Basin: K2, K3, K9

Craticula subminuscula (Manguin) C.E.Wetzel \& L.Ector (Figure 13)

Basionym: Navicula subminuscula Manguin

Ref: Lange-Bertalot 2001 p.202-pl.42: 45-50

Dimensions: Valve 7.9-11.4 $\mu \mathrm{m}$ length, 3.1-3.6 $\mu \mathrm{m}$ width and 20-22 striae in $10 \mu \mathrm{m}$

Distribution in Küçük Menderes River Basin: K1, K2, K3, K5, K9, K10

Cyclostephanos dubius (Hustedt) Round (Figure 14)

Basionym: Stephanodiscus dubius Hustedt

Ref: Krammer \& Lange-Bertalot 1991 p. 64-pl.67:8a-9b; Wojtal \& Kwandrans 2006 p.196-pl.15: 8, 16: 1-11

Dimensions: Valve diameter 9.9-11.2 $\mu \mathrm{m}$. The valve has 8-11 interstriae in $10 \mu \mathrm{m}$

Distribution in Küçük Menderes River Basin: K6, K9, K10 


\section{Cyclostephanos invisitatus (Hohn \& Hellermann) Theriot,}

Stoermer \& Håkasson (Figure 15)

Basionym: Stephanodiscus invisitatus Hohn \& Hellermann

Ref: Krammer \& Lange-Bertalot 1991 p.63-pl.67: 3, 4; Wojtal \& Kwandrans 2006 p.198-pl.15: 9, 16: 12-14, 17

Dimensions: Valve diameter 9.8-11.3 $\mu \mathrm{m}$. The valve has 9-13 interstriae in $10 \mu \mathrm{m}$

Distribution in Küçük Menderes River Basin: K10

\section{Cyclotella atomus Hustedt (Figure 16)}

Ref: Krammer \& Lange-Bertalot 1991 p.53-pl.51: 19-21; Wojtal \& Kwandrans 2006 p.184-pl.4: 13-15, 6:1-6

Dimensions: Valve diameter 6.1-12.4 $\mu \mathrm{m}$. There are 16-19 interstriae in $10 \mu \mathrm{m}$

Distribution in Küçük Menderes River Basin: K2

Cyclotella meneghiniana Kützing (Figure 17)

Ref: Krammer \& Lange-Bertalot 1991 p. 44-pl.44: 1-10; Wojtal \& Kwandrans 2006 p.186-pl.4: 18-21, 7: 1-13, 9:1-8, 10:1-5

Dimensions: Valve diameter 11.3-15.4 $\mu \mathrm{m}$. There are 6-8 interstriae in $10 \mu \mathrm{m}$

Distribution in Küçük Menderes River Basin: K3, K5, K7

Cymbella cymbiformis C. Agardh (Figure 18)

Ref: Hofmann et al. 2011 p.148-pl.80: 1-11

Dimensions: Valve 47.8-75.4 $\mu \mathrm{m}$ length, 13.5-15.4 $\mu \mathrm{m}$ width and 8-9 striae in $10 \mu \mathrm{m}$

Distribution in Küçük Menderes River Basin: K8

Cymbella excisa Kützing (Figure 19)

Ref: Hofmann et al. 2011 p.150-pl.77: 23-28

Dimensions: Valve 19.8-34.5 $\mu \mathrm{m}$ length, 7.5-9.8 $\mu \mathrm{m}$ width and 9-12 striae in $10 \mu \mathrm{m}$

Distribution in Küçük Menderes River Basin: K8, K9

Cymbella lange-bertalotii Krammer (Figure 20)

Ref: Hofmann et al. 2011 p.154-pl.78: 1-5

Dimensions: Valve 50.7-71.6 $\mu \mathrm{m}$ length, 11.2-14.5 $\mu \mathrm{m}$ width and 9-11 striae in $10 \mu \mathrm{m}$ Distribution in Küçük Menderes River Basin: K8

Cymbella parva (W. Smith) Cleve (Figure 21)

Basionym: Cocconema parvum W.Smith

Ref: Hofmann et al. 2011 p.156-pl.77: 29-34

Dimensions: Valve 35.4-39.5 $\mu \mathrm{m}$ length, 8.5-9.7 $\mu \mathrm{m}$ width and 10-11 striae in $10 \mu \mathrm{m}$

Distribution in Küçük Menderes River Basin: K7 
Cymbopleura amphicephala Näegeli (Figure 22)

Ref: Hofmann et al. 2011 p.160-pl.83: 11-15

Dimensions: Valve 24.1-31.5 $\mu \mathrm{m}$ length, 7.9-8.3 $\mu \mathrm{m}$ width and 13-15 striae in $10 \mu \mathrm{m}$ Distribution in Küçük Menderes River Basin: K7

Diatoma moniliformis (Kützing) D.M.Williams (Figure 23)

Basionym: Diatoma tenue var. moniliforme Kützing

Ref: Hofmann et al. 2011 p.174-pl.2: 11-15

Dimension: Valve 18.4-27.8 $\mu \mathrm{m}$ length, 3.5-3.9 $\mu \mathrm{m}$ width and 6-9 transapical partitions in $10 \mu \mathrm{m}$

Distribution in Küçük Menderes River Basin: K4, K5

Encyonema caespitosum Kützing (Figure 24)

Ref: Hofmann et al. 2011 p.186-pl.86: 5-9

Dimension: Valve 25.4-32.8 $\mu \mathrm{m}$ length, 8.3-11.5 $\mu \mathrm{m}$ width and 10-11 striae in $10 \mu \mathrm{m}$. Distribution in Küçük Menderes River Basin: K8.

Encyonema ventricosum (C.Agardh) Grunow (Figure 25)

Basionym: Frustulia ventricosa C.Agardh

Ref. Hofmann et al. 2011 p.192-pl.87: 18-22

Dimensions: Valve 15.4-21.2 $\mu \mathrm{m}$ length, 6.8-7.3 $\mu \mathrm{m}$ width and 12-15 striae in $10 \mu \mathrm{m}$ Distribution in Küçük Menderes River Basin: K1, K6, K7

Encyonopsis microcephala (Grunow) Krammer (Figure 26)

Ref: Hofmann et al. 2011 p.197-pl.89: 35-39

Dimensions: Valve 13.1-16.7 $\mu \mathrm{m}$ length, 3.0-3.7 $\mu \mathrm{m}$ width and 24-25 striae in $10 \mu \mathrm{m}$ Distribution in Küçük Menderes River Basin: K6, K7

\section{Encyonopsis minuta Krammer \& Reichardt (Figure 27)}

Ref: Hofmann et al. 2011 p.198-pl.89: 25-34

Dimensions: Valve 9.3-15.2 $\mu \mathrm{m}$ length, 2.9-3.8 $\mu \mathrm{m}$ width and 24-25 striae in $10 \mu \mathrm{m}$

Distribution in Küçük Menderes River Basin: K6, K7, K8

Encyonopsis subminuta Krammer \& Reichardt (Figure 28)

Ref: Hofmann et al. 2011 p.198-p1.89: 17-21

Dimensions: Valve 14.3-21.4 $\mu \mathrm{m}$ length, 3.6-4.1 $\mu \mathrm{m}$ width and 24-26 striae in $10 \mu \mathrm{m}$

Distribution in Küçük Menderes River Basin: K6, K7, K8, K10 
Fallacia pygmaea (Kützing) Stikle et Mann (Figure 29)

Basionym: Navicula pygmaea Kützing

Ref: Hofmann et al. 2011 p.245-pl.46: 31-34

Dimensions: Valve 18.1-24.8 $\mu \mathrm{m}$ length, 9.2-14.6 $\mu \mathrm{m}$ width and 22-25 striae in $10 \mu \mathrm{m}$

Distribution in Küçük Menderes River Basin: K10

Fragilaria capucina var. vaucheriae (Kützing) Lange-Bertalot (Figure 30)

Basionym: Exilaria vaucheria Kützing

Ref: Hofmann et al. 2011 p.277-pl.9: 1-7

Dimensions: Valve 15.1-22.3 $\mu \mathrm{m}$ length, 4.7-5.2 $\mu \mathrm{m}$ width and 9-10 striae in $10 \mu \mathrm{m}$

Distribution in Küçük Menderes River Basin: K8, K10

Fragilaria mesolepta Rabenhorst (Figure 31)

Ref: Hofmann et al. 2011 p.267-pl.8: 22-27

Dimensions: Valve 22.3-26.7 $\mu \mathrm{m}$ length, 3.4-4.1 $\mu \mathrm{m}$ width and 16-18 striae in $10 \mu \mathrm{m}$

Distribution in Küçük Menderes River Basin: K8 

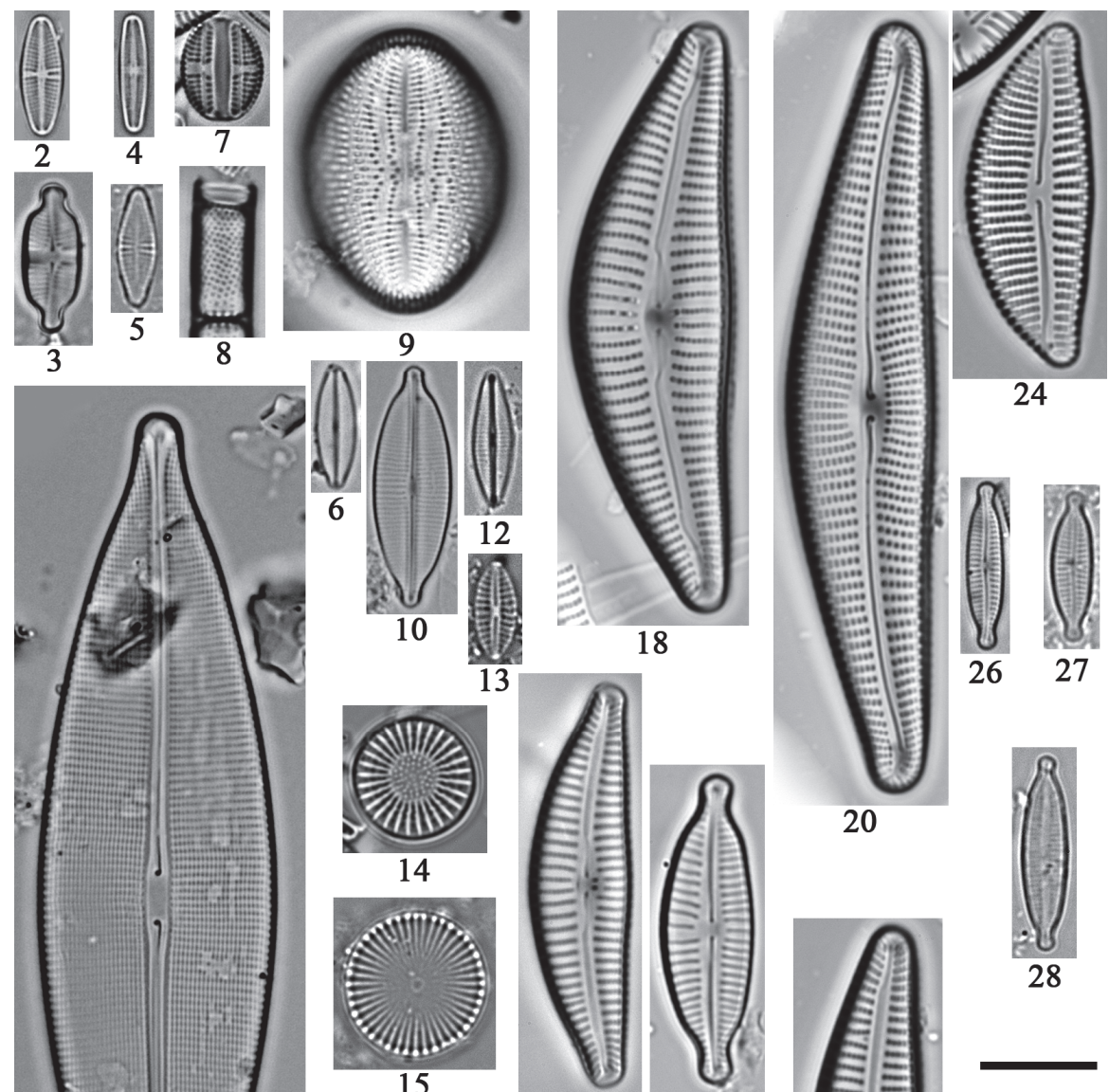

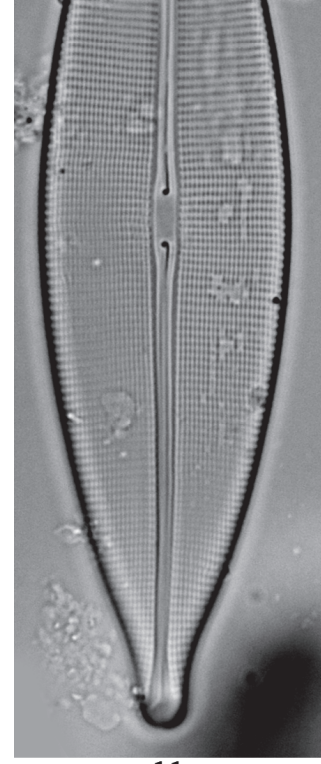

11

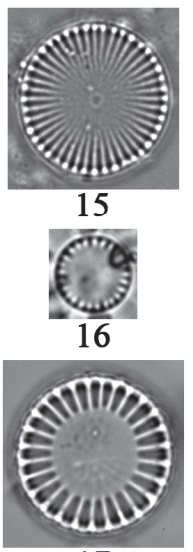

17

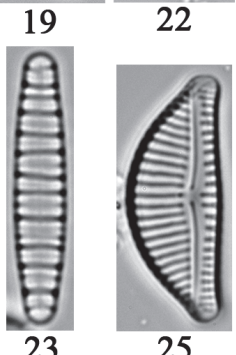

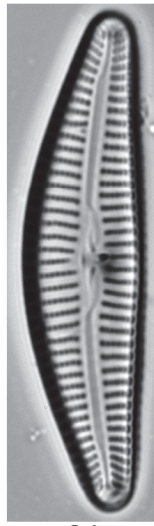

21
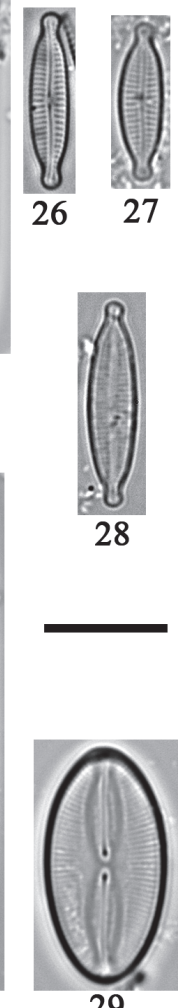

29

Figures 2-29. 2- Achnanthidium eutrophilum; 3- A. exiguum; 4- A. minutissimum; 5- A. minutissimum var. jackii; 6- Adlafia minuscula; 7- Amphora pediculus; 8- Aulacoseira ambigua; 9- Cocconeis pediculus; 10- Craticula accomoda; 11- C. cuspidata; 12- C. melostiformis; 13- C. subminuscula; 14- Cyclostephanos dubius; 15- C. invisitatus; 16- Cyclotella atomus; 17- C. meneghiniana; 18- C. cymbiformis; 19- C. excisa; 20- C. lange-bertalotii; 21- C. parva; 22- Cymbopleura amphicephala; 23- Diatoma moniliformis; 24- Encyonema caespitosum; 25- E. ventricosum; 26- Encyonopsis microcephala; 27- E. minuta Krammer \& Reichardt; 28- E. subminuta Krammer \& Reichardt; 29- Fallacia pygmaea (Kütz.) Stikle et Mann. Scale bar: $10 \mu \mathrm{m}$. 
Fragilaria perminuta (Grunow) Lange-Bertalot (Figure 32)

Ref: Hofmann et al. 2011 p.271-pl.8: 28-31

Dimensions: Valve 11.2-21.2 $\mu \mathrm{m}$ length, 2.6-3.1 $\mu \mathrm{m}$ width and 17-18 striae in $10 \mu \mathrm{m}$

Distribution in Küçük Menderes River Basin: K8, K9

Geissleria decussis (Østrup) Lange-Bertalot \& Metzeltin (Figure 33)

Basionym: Navicula decussis Østrup

Ref: Hofmann et al. 2011 p.284-pl.51: 40-44

Dimensions: Valve 18.1-23.4 $\mu \mathrm{m}$ length, 6.9-7.8 $\mu \mathrm{m}$ width and 15-16 striae in $10 \mu \mathrm{m}$

Distribution in Küçük Menderes River Basin: K1

Gomphonema olivaceum (Hornemann) Brébisson (Figure 34)

Basionym: Ulva olivacea Hornemann

Ref: Hofmann et al. 2011 p.310-pl.95: 1-6

Dimensions: Valve 14.0-21.3 $\mu \mathrm{m}$ length, 4.8-5.9 $\mu \mathrm{m}$ width and 9-12 striae in $10 \mu \mathrm{m}$

Distribution in Küçük Menderes River Basin: K7, K8

Gomphonema parvulum (Kützing) Kützing (Figure 35)

Basionym: Sphenella parvula Kützing

Ref: Hofmann et al. 2011 p.312-pl.99: 1-5

Dimensions: Valve 10.2-15.3 $\mu \mathrm{m}$ length, 4.5-4.9 $\mu \mathrm{m}$ width and 16-18 striae in $10 \mu \mathrm{m}$

Distribution in Küçük Menderes River Basin: K1, K4, K5

Gomphonema pumilum (Grunow) E.Reichardt \& Lange-Bertalot (Figure 36)

Basionym: Gomphonema intricatum var. pumilum Grunow

Ref: Hofmann et al. 2011 p.315-pl.97: 10-20

Dimensions: Valve 17.6-18.5 $\mu \mathrm{m}$ length, 4.1-4.9 $\mu \mathrm{m}$ width and 11-13 striae in $10 \mu \mathrm{m}$

Distribution in Küçük Menderes River Basin: K1, K6, K7

Halamphora montana (Krasske) Levkov (Figure 37)

Basionym: Amphora montana Krasske

Ref: Hofmann et al. 2011 p.328-p1.92: 12-14

Dimensions: Valve 14.1-16.1 $\mu \mathrm{m}$ length and 3.1-3.6 $\mu \mathrm{m}$ width

Distribution in Küçük Menderes River Basin: K3, K5, K10

Halamphora veneta (Kützing) Levkov (Figure 38)

Basionym: Amphora veneta Kützing

Ref: Hofmann et al. 2011 p.330-pl.92: 20-25

Dimensions: Valve 15.2-18.3 $\mu \mathrm{m}$ length, 4.1-4.4 $\mu \mathrm{m}$ width and 17-19 striae in $10 \mu \mathrm{m}$

Distribution in Küçük Menderes River Basin: K1, K2, K5, K9 
Hantzschia amphioxys (Ehrenberg) Grunow (Figure 39)

Basionym: Eunotia amphioxys Ehrenberg

Ref: Hofmann et al. 2011 p.333-pl.102: 1-5

Dimensions: Valve 16.1-38.4 $\mu \mathrm{m}$ length, 5.1-6.2 $\mu \mathrm{m}$ width and 19-22 striae in $10 \mu \mathrm{m}$

Distribution in Küçük Menderes River Basin: K1

Hippodonta capitata (Ehrenberg) Lange-Bertalot, Metzeltin et Witkowski (Figure 40

Basionym: Navicula capitata Ehrenberg

Ref: Hofmann et al. 2011 p.335-pl.51: 1-6

Dimensions: Valve 21.3-24.2 $\mu \mathrm{m}$ length, 5.5-6.9 $\mu \mathrm{m}$ width and 8-9 striae in $10 \mu \mathrm{m}$.

Distribution in Küçük Menderes River Basin: K10

\section{Luticola goeppertiana (Bleisch) D.G.Mann (Figure 41)}

Basionym: Stauroneis geoppertiana Bleisch

Ref: Hofmann et al. 2011 p.346-pl.45: 22-26

Dimensions: Valve 17.2-21.3 $\mu \mathrm{m}$ length, 6.8-7.3 $\mu \mathrm{m}$ width and 17-20 striae in $10 \mu \mathrm{m}$

Distribution in Küçük Menderes River Basin: K5

\section{Luticola ventricosa (Kützing) D.G.Mann (Figure 42)}

Basionym: Stauroneis ventricosa Kützing

Ref: Levkov et al. 2013 p.250-pl.190: 1-57

Dimensions: Valve 12.4-17.5 $\mu \mathrm{m}$ length, 6.2-7.1 $\mu \mathrm{m}$ width and 18-20 striae in $10 \mu \mathrm{m}$

Distribution in Küçük Menderes River Basin: K5

Mayamaea permitis (Hustedt) K.Bruder \& L.K. Medlin (Figure 43)

Basionym: Navicula permitis Hustedt

Ref: Lange-Bertalot 2001 p.136-pl.104: 7-13

Dimensions: Valve 6.5-7.3 $\mu \mathrm{m}$ length and 3.1-3.4 $\mu \mathrm{m}$ width

Distribution in Küçük Menderes River Basin: K2, K3, K4, K10

Melosira varians Agardh (Figure 44)

Ref: Krammer \& Lange-Bertalot 1991 p.7-pl.4:1-8, Wojtal 2009 p.238-pl.1: 1-4

Dimensions: Valve diameter 8.5-10.9 $\mu \mathrm{m}$

Distribution in Küçük Menderes River Basin: K10

Navicula antonii Lange-Bertalot (Figure 45)

Ref: Lange-Bertalot 2001 p.15-pl.13: 1-15

Dimensions: Valve 15.5-23.2 $\mu \mathrm{m}$ length, 5.9-6.8 $\mu \mathrm{m}$ width and 12-14 striae in $10 \mu \mathrm{m}$

Distribution in Küçük Menderes River Basin: K1, K7 
Navicula capitatoradiata Germain (Figure 46)

Ref: Lange-Bertalot 2001 p.22-pl.29: 15-20

Dimensions: Valve 30.3-35.7 $\mu \mathrm{m}$ length, 6.9-7.6 $\mu \mathrm{m}$ width and 12-14 striae in $10 \mu \mathrm{m}$

Distribution in Küçük Menderes River Basin: K4, K7, K8, K9

Navicula cryptocephala Kützing (Figure 47)

Ref: Lange-Bertalot 2001 p.27-pl.17: 1-10

Dimensions: Valve 25.4-35.2 $\mu \mathrm{m}$ length, 5.9-6.4 $\mu \mathrm{m}$ width and 15-17striae in $10 \mu \mathrm{m}$

Distribution in Küçük Menderes River Basin: K4, K7, K8, K9

Navicula cryptotenella Lange-Bertalot (Figure 48)

Ref: Lange-Bertalot 2001 p.28-pl.26: 17-32

Dimensions: Valve 18.3-27.5 $\mu \mathrm{m}$ length, 5.3-6.2 $\mu \mathrm{m}$ width and 15-17 striae in $10 \mu \mathrm{m}$ Distribution in Küçük Menderes River Basin: K9

Navicula cryptotenelloides Lange-Bertalot (Figure 49)

Ref: Lange-Bertalot 2001 p.29-pl.18: 1-8

Dimensions: Valve 12.7-21.2 $\mu \mathrm{m}$ length, 3.9-4.1 $\mu \mathrm{m}$ width and 17-18 striae in $10 \mu \mathrm{m}$

Distribution in Küçük Menderes River Basin: K8

Navicula erifuga Lange-Bertalot (Figure 50)

Ref: Hofmann et al. 2011 p.382-pl.38: 12-16

Dimensions: Valve 21.4-32.5 $\mu \mathrm{m}$ length, 5.2-6.9 $\mu \mathrm{m}$ width and 12-14 striae in $10 \mu \mathrm{m}$

Distribution in Küçük Menderes River Basin: K10

Navicula gregaria Donkin (Figure 51)

Ref: Hofmann et al. 2011 p.384-pl.39: 5-11

Dimensions: Valve 17.8-27.4 $\mu \mathrm{m}$ length, 6.1-7.2 $\mu \mathrm{m}$ width and 16-19 striae in $10 \mu \mathrm{m}$ Distribution in Küçük Menderes River Basin: K1, K2, K3, K10

Navicula notha Wallace (Figure 52)

Ref: Hofmann et al. 2011 p.390-pl.31: 19-23

Dimensions: Valve 24.2-28.4 $\mu \mathrm{m}$ length, 4.7-5.4 $\mu \mathrm{m}$ width and 16-17 striae in $10 \mu \mathrm{m}$

Distribution in Küçük Menderes River Basin: K10

Navicula radiosa Kützing (Figure 53)

Ref: Lange-Bertalot 2001 p.59-p1.8: 1-7

Dimensions: Valve 54.2-67.4 $\mu \mathrm{m}$ length, 9.1-11.4 $\mu \mathrm{m}$ width and 10-12 striae in $10 \mu \mathrm{m}$

Distribution in Küçük Menderes River Basin: K8 
Navicula reichardtiana Lange-Bertalot (Figure 54)

Ref: Lange-Bertalot 2001 p.63-pl.13: 13-25

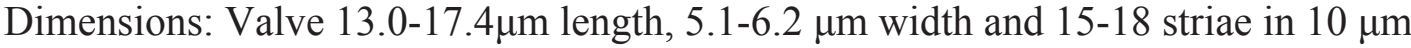

Distribution in Küçük Menderes River Basin: K1

Navicula simulata Manguin (Figure 55)

Ref: Hofmann et al. 2011 p.400-pl.38: 6-11

Dimensions: Valve 30.2-35.4 $\mu \mathrm{m}$ length, 6.2-7.0 $\mu \mathrm{m}$ width and 14-16 striae in $10 \mu \mathrm{m}$

Distribution in Küçük Menderes River Basin: K2, K9

Navicula trivialis Lange-Bertalot (Figure 56)

Ref: Lange-Bertalot 2001 p.73-pl.29: 1-6

Dimensions: Valve 32.8-42.4 $\mu \mathrm{m}$ length, 9.3-11.4 $\mu \mathrm{m}$ width and 11-12 striae in $10 \mu \mathrm{m}$ Distribution in Küçük Menderes River Basin: K9

Navicula vandamii Schoeman \& Archibald (Figure 57)

Ref: Hofmann et al. 2011 p.406-pl.29: 15-19

Dimensions: Valve 19.4-25.9 $\mu \mathrm{m}$ length, 4.7-5.1 $\mu \mathrm{m}$ width and 15-16 striae in $10 \mu \mathrm{m}$

Distribution in Küçük Menderes River Basin: K2, K9

Navicula veneta Kützing (Figure 58)

Ref: Lange-Bertalot 2001 p.78-pl.14: 23-30

Dimensions: Valve 15.4-21.9 $\mu \mathrm{m}$ length, 4.9-5.7 $\mu \mathrm{m}$ width and 14-15 striae in $10 \mu \mathrm{m}$ Distribution in Küçük Menderes River Basin: K1, K2, K4, K5, K6, K10

Navicula viridula var. germainii (Wallace) Lange-Bertalot (Figure 59)

Basionym: Navicula germainii Wallace

Ref: Hofmann et al. 2011 p.383-pl.37: 5-9

Dimensions: Valve 28.7-34.3 $\mu \mathrm{m}$ length, 6.9-7.8 $\mu \mathrm{m}$ width and 13-14 striae in $10 \mu \mathrm{m}$ Distribution in Küçük Menderes River Basin: K2, K10

Navicula viridula var. rostellata (Kützing) Cleve (Figure 60)

Basionym: Navicula rostellata Kützing

Ref: Hofmann et al. 2011 p.397-pl.37: 10-14

Dimensions: Valve 37.9-42.5 $\mu \mathrm{m}$ length, 8.7-9.9 $\mu \mathrm{m}$ width and 11-12 striae in $10 \mu \mathrm{m}$

Distribution in Küçük Menderes River Basin: K10 
Nitzschia amphibia Grunow (Figure 61)

Ref: Hofmann et al. 2011 p.433-pl.117: 9-15

Dimensions: Valve 21.5-27.4 $\mu \mathrm{m}$ length, 3.9-4.7 $\mu \mathrm{m}$ width and 15-17 striae and 7-8 fibulae in $10 \mu \mathrm{m}$

Distribution in Küçük Menderes River Basin: K4, K5

Nitzschia archibaldii Lange-Bertalot (Figure 62)

Ref: Hofmann et al. 2011 p.438-pl.113: 11-16

Dimensions: Valve 14.7-25.4 $\mu \mathrm{m}$ length, 1.8-2.3 $\mu \mathrm{m}$ width and 16-19 striae and 7-8 fibulae in $10 \mu \mathrm{m}$

Distribution in Küçük Menderes River Basin: K2, K3, K10

Nitzschia capitellata Hust. in A. Schmidt (Figure 63)

Ref: Hofmann et al. 2011 p.438-pl.113: 11-16

Dimensions: Valve 37.4-45.9 $\mu \mathrm{m}$ length, 3.9-5.1 $\mu \mathrm{m}$ width and 10-14 fibulae in $10 \mu \mathrm{m}$

Distribution in Küçük Menderes River Basin: K2, K3, K4, K10

Nitzschia desertorum Hustedt (Figure 64)

Ref: Krammer \& Lange-Bertalot 1988 p.98-pl.70: 10-13

Dimensions: Valve 16.4-17.1 $4 \mu \mathrm{m}$ length, 3.7-4.3 $\mu \mathrm{m}$ width and 25-26 striae and 1415 fibulae in $10 \mu \mathrm{m}$

Distribution in Küçük Menderes River Basin: K2

Nitzschia dissipata (Kützing) Grunow (Figure 65)

Ref: Hofmann et al. 2011 p.442-pl.109: 8-13

Dimension: Valve 13.4-23.2 $\mu \mathrm{m}$ length, 3.3-4.7 $\mu \mathrm{m}$ width and 8-10 fibulae in $10 \mu \mathrm{m}$

Distribution in Küçük Menderes River Basin: K9

Nitzschia dissipata var. media (Hantzsch) Grunow (Figure 66)

Basionym: Nitzschia media Hantzsch

Ref: Hofmann et al. 2011 p.442-pl.109: 14-18

Dimensions: Valve 47.2-61.5 $\mu \mathrm{m}$ length, 4.2-5.1 $\mu \mathrm{m}$ width and 8-10 fibulae in $10 \mu \mathrm{m}$

Distribution in Küçük Menderes River Basin: K9 


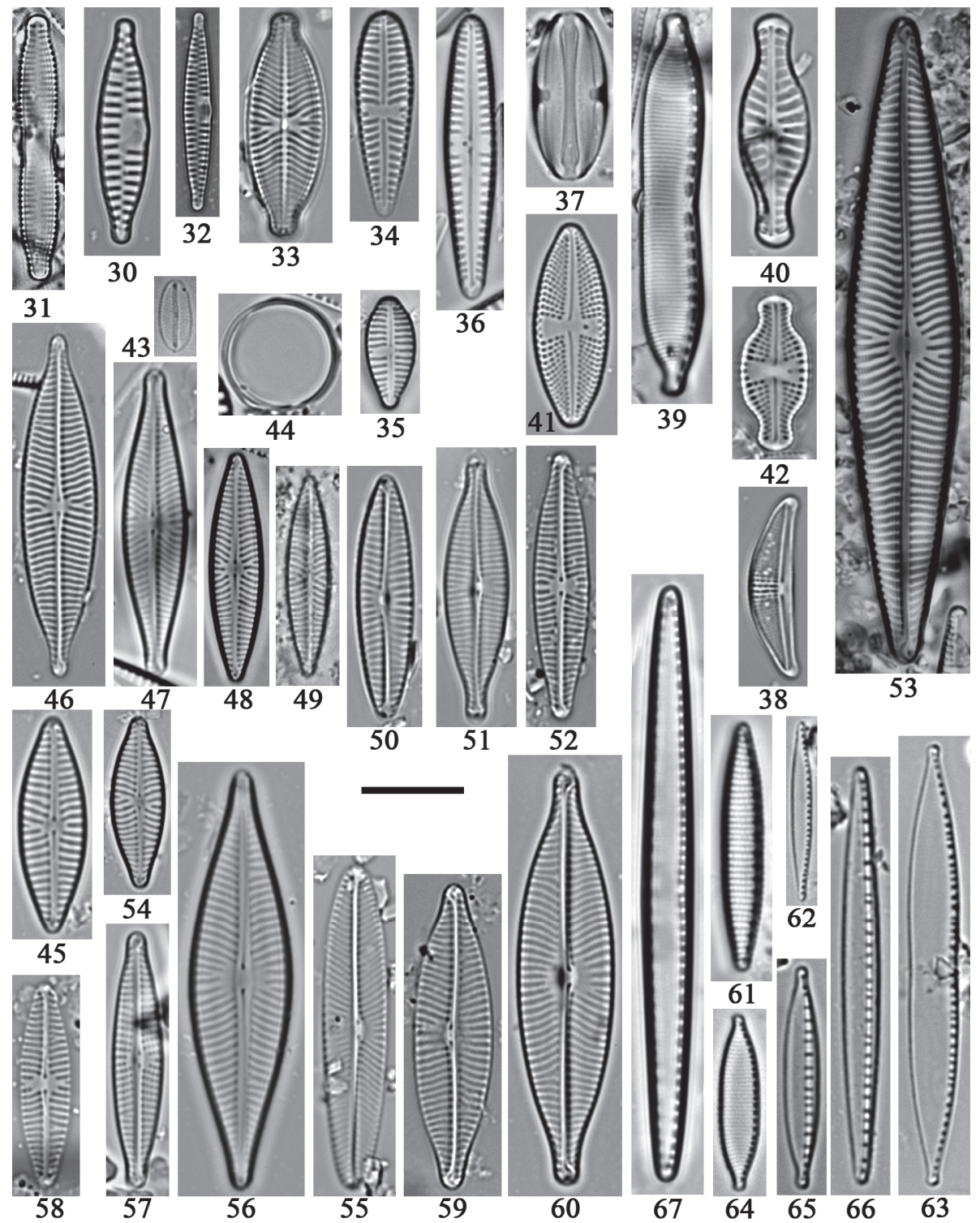

Figures 30-67. 30- Fragilaria capucina vaucheriae; 31-F. mesolepta; 32- F. perminuta; 33- Geissleria decussis; 34- Gomphonema olivaceum; 35- G. parvulum; 36- G. pumilum; 37- Halamphora montana; 38- H. veneta; 39- Hantzschia amphioxys; 40- Hippodonta capitata; 41- Luticola geoppertiana; 42- L. ventricosa; 43- Mayamaea permitis; 44- Melosira varians Agardh; 45- Navicula antonii; 46- N. capitatoradiata; 47- N. cryptocephala; 48- N. cryptotenella; 49- N. cryptotenelloides; 50- N. erifuga; 51- N. gregaria; 52- N. notha; 53- N. radiosa; 54- N. reichardtiana; 55- N. simulata; 56- N. trivialis; 57- N. vandamii; 58- N. veneta; 59- N. viridula var. germainii; 60- N. viridula var. rostellata; 61- Nitzschia amphibia; 62- N. archibaldii; 63- N. capitellata; 64- N. desertorum;

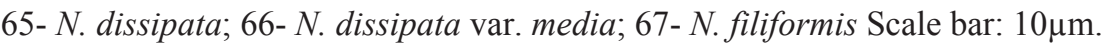


Nitzschia filiformis (W.Smith) Van Heurck (Figure 67)

Basionym: Homoecladia filiformis W.Smith

Ref: Hofmann et al. 2011 p.443-pl.116: 1-6

Dimensions: Valve 48.4-61.7 $\mu \mathrm{m}$ length, 4.5-5.7 $\mu \mathrm{m}$ width and 8-10 fibulae in $10 \mu \mathrm{m}$

Distribution in Küçük Menderes River Basin: K9

Nitzschia fonticola (Grunow) Grunow in Van Heurck (Figure 68)

Basionym: Nitzschia palea var. fonticola Grunow

Ref: Hofmann et al. 2011 p.444-pl.108: 9-15

Dimensions: Valve 27.2-35.4 $\mu \mathrm{m}$ length, 4.0-4.9 $\mu \mathrm{m}$ width and 24-28 striae in $10 \mu \mathrm{m}$

Distribution in Küçük Menderes River Basin: K1, K3

Nitzschia frustulum (Kützing) Grunow (Figure 69)

Basionym: Synedra frustulum Grunow

Ref: Hofmann et al. 2011 p.445-pl.112: 28-34

Dimensions: Valve 14.7-21.3 $\mu \mathrm{m}$ length, 2.9-3.5 $\mu \mathrm{m}$ width and 21-24 striae and 10-12 fibulae in $10 \mu \mathrm{m}$

Distribution in Küçük Menderes River Basin: K9, K10

Nitzschia inconspicua Grunow (Figure 70)

Ref: Hofmann et al. 2011 p.445-pl.112: 35-40

Dimensions: Valve 6.4-10.5 $\mu \mathrm{m}$ length, 2.5-2.9 $\mu \mathrm{m}$ width and 26-28 striae and 13-14 fibulae in $10 \mu \mathrm{m}$

Distribution in Küçük Menderes River Basin: K1, K4, K8, K9, K10

Nitzschia linearis (C.Agardh) W. Smith (Figure 71)

Basionym: Frustulia sociabilis C.Agardh

Ref: Hofmann et al. 2011 p.558-pl.126: 1-5

Dimensions: Valve 45.4-62.7 $\mu \mathrm{m}$ length, 5.5-6.1 $\mu \mathrm{m}$ width and 10-12 fibulae in $10 \mu \mathrm{m}$

Distribution in Küçük Menderes River Basin: K1

Nitzschia palea (Kützing) W.Smith (Figure 72)

Basionym: Synedra palea Kützing

Ref: Hofmann et al. 2011 p.454-pl.111:1-9

Dimensions: Valve 37.5-50.4 $\mu \mathrm{m}$ length, 3.6-3.9 $\mu \mathrm{m}$ width and 10-12 fibulae in $10 \mu \mathrm{m}$

Distribution in Küçük Menderes River Basin: K1, K2, K3, K4, K5, K6, K7, K10

Nitzschia sociabilis Hustedt (Figure 73)

Ref: Hofmann et al. 2011 p.461-pl.111: 35-40

Dimensions: Valve 27.3-35.4 $\mu \mathrm{m}$ length, 3.2-3.7 $\mu \mathrm{m}$ width and 10-11 fibulae in $10 \mu \mathrm{m}$

Distribution in Küçük Menderes River Basin: K9 
Nitzschia umbonata (Ehrenberg) Lange-Bertalot (Figure 74)

Basionym: Navicula umbonata Ehrenberg

Ref: Hofmann et al. 2011 p.558-pl.126: 1-5

Dimensions: Valve 45.2-65.7 $\mu \mathrm{m}$ length, 6.5-7.8 $\mu \mathrm{m}$ width and 9-10 fibulae in $10 \mu \mathrm{m}$

Distribution in Küçük Menderes River Basin: K1, K2, K4, K5, K6

Pantocsekiella ocellata (Pantocsek) K.T.Kiss \& Ács (Figure 75)

Basionym: Cyclotella ocellata Pantocsek

Ref: Krammer \& Lange-Bertalot 1991 p. 51-pl.50: 1-11; Wojtal \& Kwandrans 2006 p.188-pl.7: 26-27

Dimensions: Valve diameter 8.2-10.4 $\mu \mathrm{m}$. Valves have 16-18 interstriae in $10 \mu \mathrm{m}$ Distribution in Küçük Menderes River Basin: K8

Planothidium lanceolatum (Brébisson ex Kützing) Lange-Bertalot (Figure 76)

Basionym: Achnanthidium lanceolatum Brébisson ex Kützing

Ref: Hofmann et al. 2011 p.510-pl.24: 41-47

Dimensions: Valve 15.1-18.4 $\mu \mathrm{m}$ length, 4.5-7.8 $\mu \mathrm{m}$ width and 12-14 striae in $10 \mu \mathrm{m}$

Distribution in Küçük Menderes River Basin: K1, K8

Pseudofallacia monoculata (Hustedt) D.G.Mann (Figure 77)

Basionym: Navicula monoculata Hustedt

Ref: Hofmann et al. 2011 p.244-pl.46: 13-17

Dimensions: Valve 9.2-13.5 $\mu \mathrm{m}$ length, 4.2-5.1 $\mu \mathrm{m}$ width and 21-25 striae in $10 \mu \mathrm{m}$

Distribution in Küçük Menderes River Basin: K3, K10

Pseudostaurosira brevistriata (Grunow) D.M.Williams \& Round (Figure 78)

Basionym: Fragilaria brevistriata Grunow

Ref: Hofmann et al. 2011 p.258-pl.9: 25-29

Dimensions: Valve 13.2-17.8 $\mu \mathrm{m}$ length, 3.9-4.8 $\mu \mathrm{m}$ width and 14-16 striae in $10 \mu \mathrm{m}$

Distribution in Küçük Menderes River Basin: K8

Reimeria sinuata (Gregory) Kociolek et Stoermer (Figure 79)

Basionym: Cymbella sinuata Gregory

Ref: Hofmann et al. 2011 p.526-pl.89: 50-61

Dimensions: Valve 12.5-15.4 $\mu \mathrm{m}$ length, 3.9-4.7 $\mu \mathrm{m}$ width and 12-15 striae in $10 \mu \mathrm{m}$ Distribution in Küçük Menderes River Basin: K1, K2, K7 
Sellaphora pupula (Kützing) Mereschkovsky (Figure 80)

Basionym: Navicula pupula Kützing

Ref: Hofmann et al. 2011 p.536-pl.41: 1, 2

Dimensions: Valve 21.4-27.5 $\mu \mathrm{m}$ length, 6.7-7.8 $\mu \mathrm{m}$ width and 20-24 striae in $10 \mu \mathrm{m}$

Distribution in Küçük Menderes River Basin: K1, K2, K3, K10

\section{Sellaphora radiosa (Hustedt) H.Kobayasi (Figure 81)}

Basionym: Navicula seminulum var. radiosa Hustedt

Ref: Hofmann et al. 2011 p.533-p1.42: 27-31

Dimensions: Valve 10.5-13.7 $\mu \mathrm{m}$ length, 3.5-3.9 $\mu \mathrm{m}$ width and 19-21 striae in $10 \mu \mathrm{m}$

Distribution in Küçük Menderes River Basin: K5

Sellaphora saprotolerans Lange-Bertalot, Hofmann \& Cantonati (Figure 82)

Ref: Hofmann et al. 2011 p.536-pl.41: 6-10

Dimensions: Valve 27.2-28.4 $\mu \mathrm{m}$ length, 8.2-8.4 $\mu \mathrm{m}$ width and 18 striae in $10 \mu \mathrm{m}$

Distribution in Küçük Menderes River Basin: K1, K2, K10

\section{Sellaphora saugerresii (Desmazières) C.E.Wetzel \& D.G.Mann (Figure 83)}

Basionym: Navicula suagerresii Desmazières

Ref: Hofmann et al. 2011 p.201-pl.42: 37-43

Dimensions: Valve 4.5-10.8 $\mu \mathrm{m}$ length, 2.5-3.2 $\mu \mathrm{m}$ width and 26-32 striae in $10 \mu \mathrm{m}$

Distribution in Küçük Menderes River Basin: K1, K2, K3, K4

\section{Sellaphora seminulum (Grunow) D.G.Mann (Figure 84)}

Basionym: Navicula seminulum Grunow

Ref: Hofmann et al. 2011 p.537-pl.42: 22-26

Dimensions: Valve 9.2-12.5 $\mu \mathrm{m}$ length, 3.5-4.2 $\mu \mathrm{m}$ width and 19-21 striae in $10 \mu \mathrm{m}$

Distribution in Küçük Menderes River Basin: K4, K5

\section{Stephanodiscus hantzschii Grunow (Figure 85)}

Ref: Krammer \& Lange-Bertalot 1991 p.73-pl.74:12-16, 75:4-11; Wojtal \& Kwandrans 2006 p.199-pl.18: 3-8, 19: 1-9

Dimensions: Valve diameter 7.4-12.3 $\mu \mathrm{m}$. There are 9-11 interstriae in $10 \mu \mathrm{m}$

Distribution in Küçük Menderes River Basin: K10

\section{Surirella brebissonii Krammer et Lange-Bertalot (Figure 86)}

Ref: Hofmann et al. 2011 p.556-pl.130: 11-16

Dimensions: Valve 19.2-25.4 $\mu \mathrm{m}$ length, 13.2-15.4 $\mu \mathrm{m}$ width and 4-5 fibulae in $10 \mu \mathrm{m}$

Distribution in Küçük Menderes River Basin: K1, K2, K5 


\section{Surirella minuta Brébisson (Figure 87)}

Ref: Hofmann et al. 2011 p.558-pl.131: 6-12

Dimensions: Valve 25.4-29.3 $\mu \mathrm{m}$ length, 9.2-10.8 $\mu \mathrm{m}$ width and 6-7 fibulae in $10 \mu \mathrm{m}$ Distribution in Küçük Menderes River Basin: K2

\section{Surirella robusta Ehrenberg (Figure 88)}

Ref: Hofmann et al. 2011 p.559-pl.127: 1, 2

Dimensions: Valve $98.8 \mu \mathrm{m}$ length, $31.3 \mu \mathrm{m}$ width and 3 fibulae in $10 \mu \mathrm{m}$

Distribution in Küçük Menderes River Basin: K1, K2, K10

\section{Tryblionella apiculata Gregory (Figure 89)}

Ref: Hofmann et al. 2011 p.439-pl.104: 18-22

Dimensions: Valve 32.4-41.3 $\mu \mathrm{m}$ length, 4.9-6.1 $\mu \mathrm{m}$ width and 15-18 striae in $10 \mu \mathrm{m}$. Distribution in Küçük Menderes River Basin: K2

\section{Tryblionella calida (Grunow) D.G.Mann (Figure 90)}

Basionym: Nitzschia calida Grunow

Ref: Hofmann et al. 2011 p.437-pl.103: 5-8

Dimensions: Valve 34.3-37.5 $\mu \mathrm{m}$ length, 6.2-7.4 $\mu \mathrm{m}$ width and 12 fibulae in $10 \mu \mathrm{m}$ Distribution in Küçük Menderes River Basin: K2 


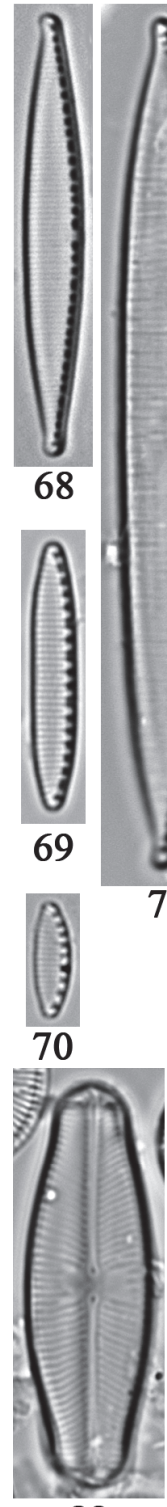

82
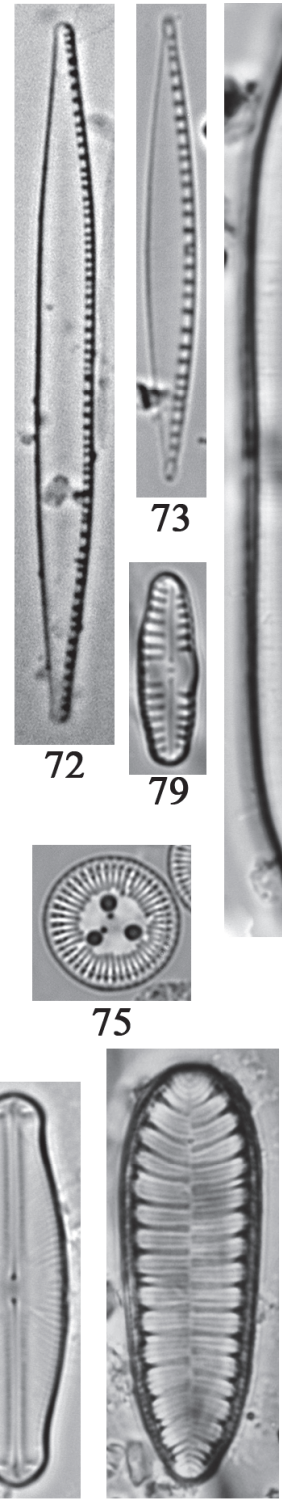

87

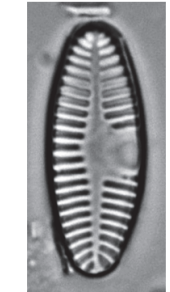

76
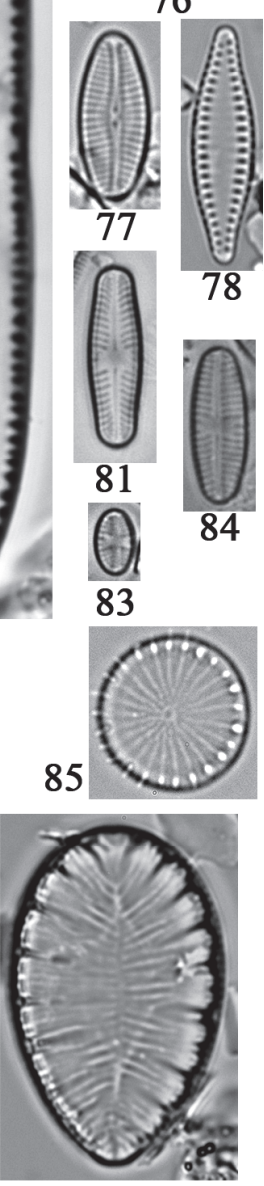

86

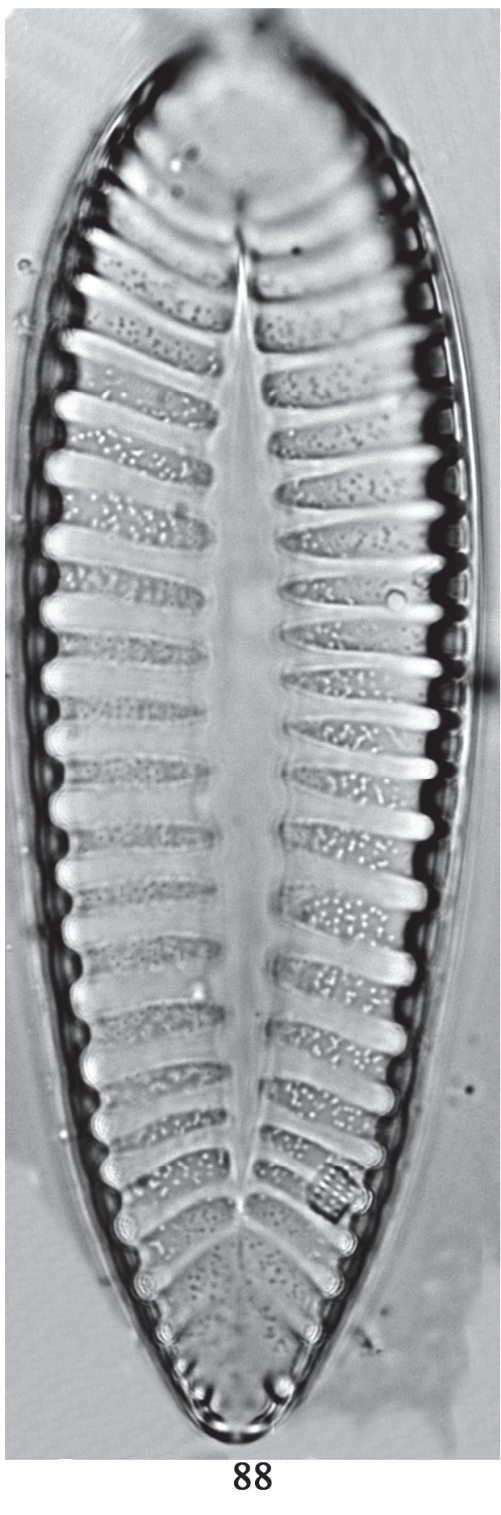

88

Figures 68-88. 68- Nitzschia fonticola; 69- N. frustulum; 70- N. inconspicua; 71- N. linearis; 72- Nitzschia palea; 73- N. sociabilis; 74- N. umbonata; 75- Pantocsekiella ocellata; 76- Planothidium lanceolatum; 77- Pseudofallacia monoculata; 78- Pseudostaurosira brevistriata; 79- Reimeria sinuata; 80- Sellaphora pupula; 81- S. radiosa; 82- S. saprotolerans; 83- S. saugerresii; 84- S. seminulum; 85- Stephanodiscus hantzschii; 86- Surirella brebissonii; 87- S. minuta; 88- S. robusta. Scale bar: $10 \mu \mathrm{m}$. 
Ulnaria acus (Nitzsch) P.Compère (Figure 91)

Basionym: Bacillaria ulna Nitzsch

Ref: Hofmann et al. 2011 p.256-pl.5: 1-5

Dimensions: Valve 80.5-92.3 $\mu \mathrm{m}$ length, 3.9-4.2 $\mu \mathrm{m}$ width and 13-15 striae in $10 \mu \mathrm{m}$

Distribution in Küçük Menderes River Basin: K8

Ulnaria biceps (Kützing) P.Compère (Figure 92)

Basionym: Synedra biceps Kützing

Ref: Krammer \& Lange-Bertalot 1991 p.146-pl.121: 1-5

Dimensions: Valve 7.3-7.9 $\mu \mathrm{m}$ width and 8-9 striae in $10 \mu \mathrm{m}$

Distribution in Küçük Menderes River Basin: K5, K7, K8

Ulnaria capitata (Ehrenberg) Compère (Figure 93)

Basionym: Synedra capitata Ehrenberg

Ref: Hofmann et al. 2011 p.277-pl.6: 1-3

Dimensions: Valve 7.2-7.6 $\mu \mathrm{m}$ width and 8-10 striae in $10 \mu \mathrm{m}$

Distribution in Küçük Menderes River Basin: K8

Ulnaria delicatissima (W.Smith) M.Aboal \& P.C.Silva (Figure 94)

Basionym: Synedra delicatissima W.Smith

Ref: Hofmann et al. 2011 p.262-pl.7: 1-6

Dimensions: Valve 47.2-58.4 $\mu \mathrm{m}$ length, 2.3-3.1 $\mu \mathrm{m}$ width and 14-15 striae in $10 \mu \mathrm{m}$

Distribution in Küçük Menderes River Basin: K6, K8

Ulnaria ulna (Nitzsch) P.Compère (Figure 95)

Basionym: Bacillaria ulna Nitzsch

Ref: Hofmann et al. 2011 p.276-pl.5: 6-11

Dimensions: Valve 64.3-82.4 $\mu \mathrm{m}$ length, 5.7-6.5 $\mu \mathrm{m}$ width and 10-12 in $10 \mu \mathrm{m}$

Distribution in Küçük Menderes River Basin: K1 
Among the taxa, Nitzschia palea was abundant in K1 and K3 stations; Sellaphora pupula was abundant is K2 station; Navicula veneta was abundant in K4; Craticula accomoda and $C$. subminuscula were abundant in K5 station; Achnanthidium minutissimum was abundant in K7; Ulnaria delicatissima was abundant in K8; Cymbella excisa was abundant in K9, Melosira varians and Nitzschia frustulum were abundant in K10.
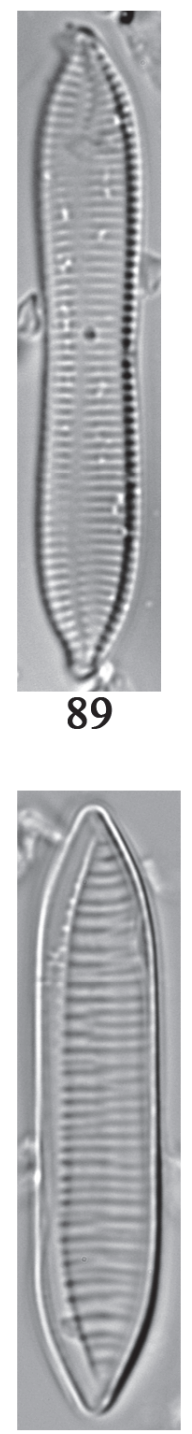

90

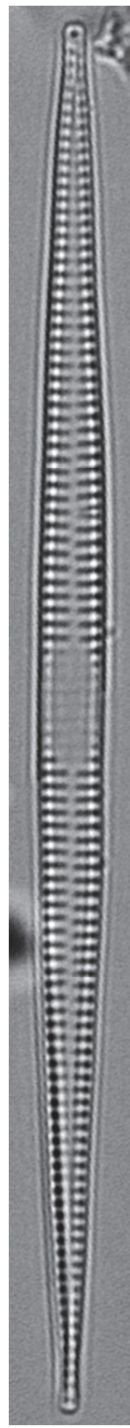

91
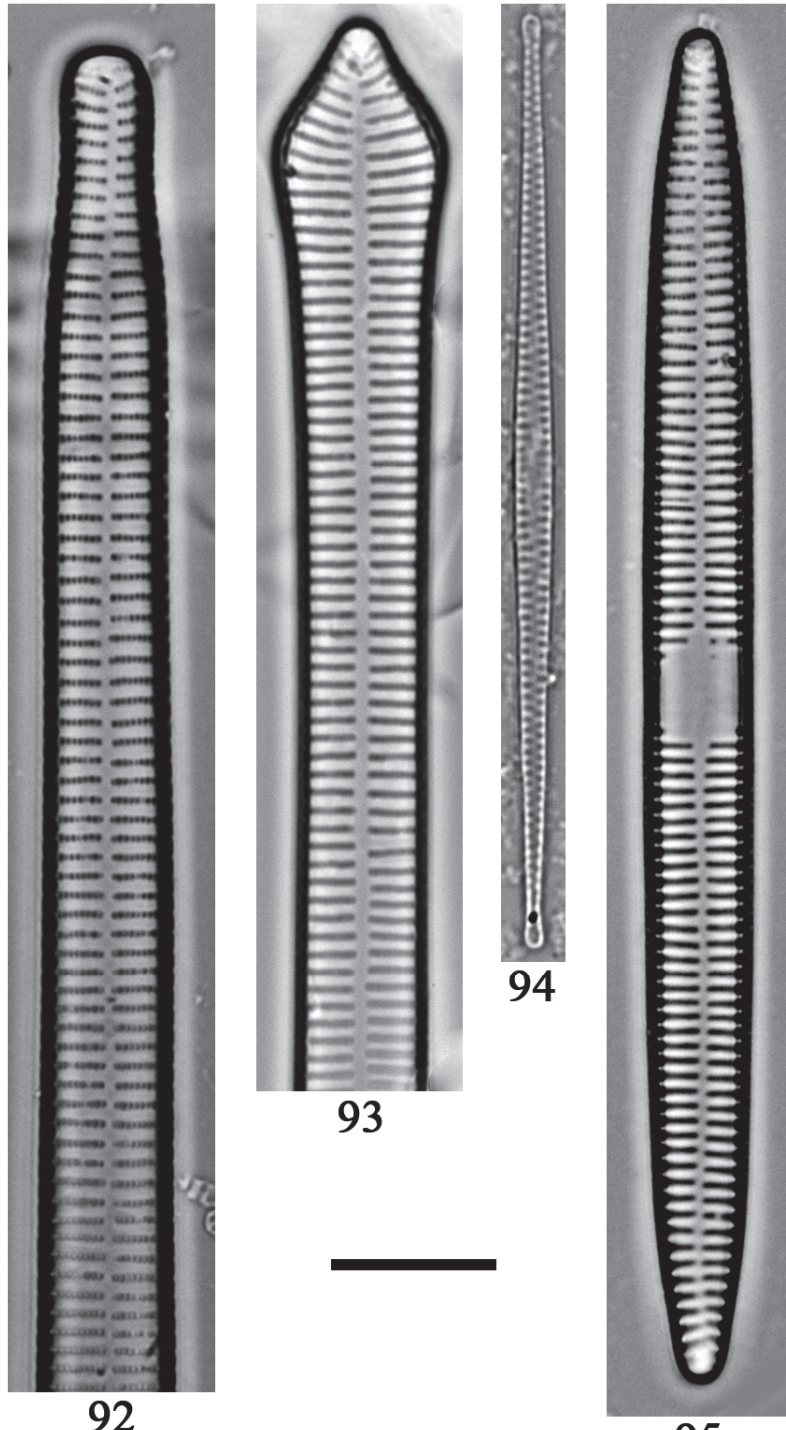

95

Figures 89-95. 89- Tryblionella apiculata; 90- T. calida; 91- Ulnaria acus; 92- U. biceps; 93- U. capitata; 94- U. delicatissima; 95- U. ulna. Scale bar: $10 \mu \mathrm{m}$. 
Regarding to the ecological status of the water bodies investigated in this study, abundant taxa like Craticula accomoda, Navicula veneta or Nitzschia palea were found as good indicators for polluted waters (Lange-Bertalot et al. 2017) and the presence of these taxa indicated that the most of the sampling stations were affected by pollution.

On the other hand, there are some differences between Turkish inland waters and water bodies in this study in terms of the distribution of the taxa. Craticula melostiformis, Cyclostephanos dubius, Encyonopsis minuta, E. subminuta, Gomphonema pumilum, Halamphora montana and Mayameae permitis were rarely found in Turkey (Gönülol, 2017) but commonly encountered in this study. Similarly, Craticula melostiformis, Gomphonema pumilum and Mayamaea permitis were newly recorded for Turkish freshwater diatom flora. The results point out that the taxa mentioned above might be common in Turkish waters but further studies might be needed to present the distribution of these taxa in Turkish freshwaters in detail and reveal the big picture.

In Turkey, common diatoms are given in the articles in the studies. However, hardly any results for rare taxa can be found in the articles. On the other hand, new records and rare species are important to observe the community structure and flora of a country and to uncover the potential indicator values of the species for monitoring studies (Potapova and Charles, 2004). In this regard, some taxa were found as new records for Turkish diatom flora namely Achnanthidium eutrophilum, $A$. minutissimum var. jackii, Navicula simulata, N. vandamii, Nitzschia archibaldii, $N$. desertorum and Sellaphora saprotolerans.

Biological monitoring studies that carried out in different river basins of Turkey are important in terms of ecosystem based evaluation of water quality. On the other hand, these studies make a great contribution to flora and fauna studies. Biological, physicochemical and chemical monitoring is carried out simultaneously in many water bodies which have not been monitored until today and getting information on the distribution and environmental needs of the species. Establishment of visual catalogues of the species which including information about dimensions and distrubiton for each river basin will greatly contribute to monitoring studies. In this way, identification process will be easier and ecological status of the water bodies can be assessed more quickly. 


\section{Discussion and Conclusion}

In this study, 94 taxa were identified in total. Among the taxa; Achnanthidium minutissimum, Adlafia minuscula, Amphora pediculus, Cocconeis pediculus, Craticula accomoda, C. cuspidata, C. subminuscula, Cyclotella meneghiniana, Cymbella cymbiformis, C. parva, Cymbopleura amphicephala, Diatoma moniliformis, Encyonema caespitosum, E. ventricosum, Encyonopsis microcephala, Fallacia pygmeae, Fragilaria capucina var. vaucheria, F. mesolepta, Geissleria decussis, Gomphonema olivaceum, G. parvulum, Halamphora veneta, Hantzschia amphioxys, Hippodonta capitata, Melosira varians, Navicula capitatoradiata, N. cryptocephala, $N$. cryptotenella, $N$. gregaria, $N$. radiosa, $N$. trivialis, $N$. veneta, $N$. viridula var. rostellata, Nitzschia amphibia, $N$. capitellata, $N$. dissipata, $N$. dissipata var. media, $N$. filiformis, $N$. fonticola, N. frustulum, $N$. inconspicua, $N$. linearis, $N$. palea, $N$. umbonata, Pantocsekiella ocellata, Planothidium lanceolatum, Pseudostaurosira brevistriata, Reimeria sinuata, Sellaphora pupula, S. saugerresii, Stephanodiscus hantzschii, Surirella brebissonii, S. minuta, S. robusta, Tryblionella apiculata, Ulnaria acus, U. biceps, U. capitata, U. delicatissima and Ulnaria ulna were common taxa in Turkish diatom flora (Gönülol, 2017).

\section{Acknowledgement}

This study was conducted considering the outcomes of "Project of Basin Monitoring and Determination of Reference Points". The authors thank to the contractors of the project and SEGAL (Environmental Measurement and Analysis Laboratory), and all the experts and academicians involved in this project for their valuable supports. 


\section{References}

Anonymous (2017). Türkiye'de referans izleme ağının kurulması projesi. Orman ve Su İsleri Bakanlı̆ Su Yönetimi Genel Müdürlüğü.

Anonymous (2016). Ülkemize özgü su kalitesi ekolojik değerlendirme sisteminin kurulması projesi. Orman ve Su İ̧sleri Bakanlı̆̆l, Su Yönetimi Genel Müdürlüğü.

Anonymous (2014a). Havza izleme ve referans noktaların belirlenmesi projesi. (Küçük Menderes, Kızılırmak, Marmara, Konya, Antalya havzalarında Su Çerçeve Direktifi kapsamında su kalitesi izleme projesi). Orman ve Su Işsleri Bakanlığı, Su Yönetimi Genel Müdürlüğü.

Anonymous (2014b). Yüzeysel Sular ve Yeraltı Sularının İzlenmesine Dair Yönetmelik. 11.02.2014, 28910. Orman ve Su Işleri Bakanlı̆̆l, Su Yönetimi Genel Müdürlüğü.

Anonymous (2014c). Havza izleme ve referans noktaların belirlenmesi projesi (Küçük Menderes havzası final raporu). Orman ve Su İsleri Bakanlı̆̆l, Su Yönetimi Genel Müdürlüğü.

Anonymous (2013). Havza izleme ve referans noktaların belirlenmesi projesi (Ergene, Akarçay, Gediz, Susurluk ve Sakarya havzalarında Su Çerçeve Direktifi kapsamında su kalitesi izleme projesi). Orman ve Su İsleri Bakanlığl, Su Yönetimi Genel Müdürlüğü.

Anonymous (2011). Orman ve Su İşleri Bakanlığının Teşkilat ve Görevleri Hakkında Kanun Hükmünde Kararname. 04.07.2011, 27984.

Anonymous (2010). EN 15708. European Committee for Standardization. Water quality - Guidance standard for the surveying, sampling and laboratory analysis of phytobenthos in shallow running water.

Anonymous (2000). Directive 2000/60/EC of the European Parliament and the council of 23 October 2000 establishing a framework for community action in the field of water policy. Official Journal of the European Communities, L327, 1-72.

Aysel, V. (2005). Check-list of the freshwater algae of Turkey. J. Black Sea/Mediterranean Environment, 11, 1-124.

Aysel, V., Gezerler-Şipal, U., Aysel, F., Erduğan, H., \& Türker, E. (2002). Barutçu Gölü’nün (Selçuk, İzmir, Türkiye) mikro-makro algleri. E. ̈̈. Su Ürünleri Dergisi, 19, 1-11.

Aysel, V., Erduğan, H., Türker, E., Aysel, F., \& Gönüz, A. (2001). Laka Deresi’nin (Bornova, İzmir, Türkiye) makro ve mikro algleri. E.Ü. Su Ürünleri Dergisi 18, 307-317.

Aysel, V., Gönüz, A., Bakan, A.N., \& Gezerler-Şipal, U. (1998). Oğlananası Gölü'nün (Gaziemir, İzmir, Türkiye) alg florası. XIV. Ulusal Biyoloji Kongresi, Samsun, 2, 254-271. 
Demir, N., Çetin, T., Gök, C., \& Şanal, M. (2017). First biological monitoring in the Akarçay basin according to the Water Framework Directive: phytoplankton and phytobenthos. Turkish Journal of Water Science and Management, 1, 90-107.

Gönülol, A. (2017). Turkish algae electronic publication. Samsun, Turkey. http://turkiyealgleri.omu.edu.tr

Gönülol, A., Öztürk, M., \& Öztürk, M. (1996). A check-list of the freshwater algae of Turkey. O.M. ̈̈. Fen Bilimleri Dergisi, 7, 8-46.

Hofmann, G., Werum, M., \& Lange-Bertalot, H. (2011). Diatomeen im Süßwasser-Benthos von Mitteleuropa. Bestimmungsflora Kieselalgen für die ökologische Praxis. Über 700 der häufigten Arten und ihre Ökologie. A.R.G. Gantner Verlag K.G., Rugell.

Krammer, K., \& Lange-Bertalot, H. (1988). Bacillariophyceae, 2. Teil: Bacillariaceae, Epithemiaceae, Surirellaceae. Süßwasserflora von Mitteleuropa, 2(2), 1-596.

Krammer, K., \& Lange-Bertalot, H. (1991). Bacillariophyceae 3.Teil: Centrales, Fragilariaceae, Eunotiaceae. Süßwasserflora von Mitteleuropa, 2(3), 1-576.

Lange-Bertalot, H., Hofmann G., Werum M., \& Cantonati M. (2017). Freshwater Benthic Diatoms of Central Europe: Over 800 Common Species Used in Ecological Assessment. A.R.G. Gantner Verlag K.G., Rugell.

Lange-Bertalot, H. (2001). Navicula sensu stricto. 10 Genera separated from Navicula sensu lato. Frustulia. Diatoms of the European inland waters and comparable habitats. Diatoms of Europe, 2, 1-526.

Levkov, Z., Metzeltin, D., \& Pavlov, A. (2013). "Luticola and Luticopsis". Diatoms of the European Inland Waters and Comparable Habitats. Diatoms of Europe, 7, 1-697.

Potapova, M., \& Charles, D.F. (2004). Potential use of rare diatoms as environmental indicators in USA rivers. - In: Poulin, M (ed.), Proceedings of the 17th International Diatom Symposium, Biopress Limited, Bristol, pp. 281-295.

Solak, C.N., Kulikovskiy, M., Kaleli, A., \& Gönülol, A. (2016). Rare and new records for diatoms of Turkey from Kütahya running waters. Oceanological \& Hydrobiological Studies, 45(4), 564-587.

Toudjani, A.A., Çelekli, A., Gümüş, E.Y., Kayhan, S., Lekesiz, H.Ö., \& Çetin, T. (2017). A new diatom index to assess ecological quality of running waters: a case study of water bodies in western Anatolia. Ann. Limnol.-Int. J. Lim. 53, 333-343.

Wojtal, A.Z. (2009). The diatoms of Kobylanka stream near Kraków (Wyżyna KrakowskoCzęstochowska, S Poland). Polish Botanical Journal, 54, 129-330.

Wojtal, A.Z., \& Kwandrans, J. (2006). Diatoms of the Wyżyna Krakowsko Częstochowska Upland (S Poland) - Coscinodiscophyceae (Thalassiosirophycidae). Polish Botanical Journal, 51, 177-207. 


\section{Extended Turkish Abstract \\ (Genişletilmiş Türkçe Özet)}

\section{Küçük Menderes Nehir Havzası’nda Bentik Diyatome (Fitobentoz) Kompozisyonunun Dağılımı}

Avrupa Birliği Su Çerçeve Direktifi'nin (2000/60/AT) (SÇD) Avrupa'da 2000 yılında yürürlüğe girmesiyle birlikte su kalitesinin geleneksel yöntemlerle fizikokimyasal ve kimyasal olarak izlenmesi anlayışı yerini biyolojik izlemenin ön planda yer aldığı ekosistem esaslı izlemeye bırakmıştır. Avrupa Birliği Üye Devletleri fitobentoz, fitoplankton, makrofit, balık, makroomurgasız, makroalg ve angiosperm gibi birçok biyolojik kalite bileşenini nehir, göl, kıyı ve geçiş suyu kütlelerinde ekosistem esaslı değerlendirme maksadıyla izlemeye başlamıştır. Fizikokimyasal ve kimyasal izleme çalışmaları ile su kütlesinin sadece ölçüm yapılan andaki kalitesi belirlenirken, biyolojik kalite bileşenleri ile yapılan izleme çalışmaları sonucunda su kütlesinin uzun süreli durumu hakkında bilgi edinilebilmektedir.

Türkiye'de Su Çerçeve Direktifi'nin uyumlaştırılması çalışmaları 2011 yılında Orman ve Su İşleri Bakanlığı Su Yönetimi Genel Müdürlüğü’nün kurulmasıyla birlikte hız kazanmıștır. Türkiye'de ilk defa SÇD kapsamında havza bazında izleme çalışmaları Su Yönetimi Genel Müdürlüğü tarafından yürütülmüş "Havza İzleme ve Referans Noktaların Belirlenmesi Projesi” ile Akarçay, Meriç-Ergene, Susurluk, Gediz ve Sakarya nehir havzalarında gerçekleştirilmiş ve ardından bu çalışmanın kaynağını oluşturan Küçük Menderes Nehir Havzası ve ayrıca Kızılırmak, Marmara, Antalya ve Konya nehir havzaları ile izleme çalışmalarına devam edilmiştir. "Ülkemize Özgü Su Kalitesi Ekolojik Değerlendirme Sisteminin Kurulması Projesi” ile Türkiye'nin farklı coğrafi ve iklim koşullarına sahip nehir havzalarında (Batı Akdeniz, Batı Karadeniz, Doğu Karadeniz, Kuzey Ege, Ceyhan, Sakarya, Aşağı Fırat ve Aras) yapılan izleme çalışmaları ile ülkemize özgü indeksler belirlenmiştir. "Türkiye'de Referans İzleme Ağı'nın Kurulması Projesi” ile Türkiye'nin 25 nehir havzasının tamamında yapılacak izlemeler ile referans izleme noktalarının belirlenmesi çalışmalarına devam edilmektedir.

Küçük Menderes Nehir Havzası, Türkiye'nin batısında Büyük Menderes ile Gediz nehir havzaları arasında yer almaktadır. Türkiye'nin yüzey alanının yaklaşık \%1'ini oluşturan havzanın sularını Ege Denizi’ne ulaştırması sebebiyle kıyı ve geçiş suyu kütleleri bulunmaktadır. SÇD kapsamında fitobentoz kalite bileşeninin sadece nehir ve göl suyu kütlelerinde izlenmekte olup, bu çalışmada Küçük Menderes Nehir Havzası'nda bulunan 7 nehir suyu kütlesi ve 3 baraj gölünde (Tahtalı, Beydağı, Alaçatı) yapılan fitobentoz izlemesi gerçekleştirilmiştir.

Fitobentoz, mikroskobik ve tek hücreli canlılardan 2 m'den uzun makrofitlere kadar tüm fototrofik canlıları kapsamaktadır. Fitobentoza yönelik pratik değerlendirme metotlarının eksikliğinden dolayı Avrupa Birliği Üye Devletleri fitobentozun en önemli göstergesi olan diyatomeleri izlemektedir. Fitobentozun en önemli göstergesi olan diyatomeler ile ilgili pek çok çalışma olmasına rağmen diyatome kompozisyonun dağılımı Türkiye'de halen yeni bir konudur. Bu çalışmada, Küçük Menderes Nehir Havzası'nda bentik diyatome kompozisyonunun dağılımının araştırılması amaçlanmış̧ır. 2014 yılı içerisinde Mayıs, Temmuz ve Eylül aylarında mevsimsel olarak epilitik ve epipelik diyatome örnekleri toplanmıştır. Epilitik diyatomeler nehir yatağında yer alan taşların firçalanmasıyla, epipelik örnekler ise pipet yardımıyla sedimentten örneklenmiştir. Diyatome früstüllerinden organik maddenin uzaklaştırılması maksadıyla $\mathrm{H}_{2} \mathrm{O}_{2}$ ve HCL kullanılmış ve Naphrax solüsyonu kullanılarak daimi preparatlar hazırlanmıştır. Diyatomeler mikroskop yardımıyla incelenmiş ve taksonomik literatüre göre teşhis edilmiştir. Her bir preparatta ortalama 400 birey sayılmıştır. Teşhis edilen türlerin uzunluk, 
genişlik ve $10 \mu \mathrm{m}$ 'deki stria sayıları raporlanmış ve türlerin görsel katalogları oluşturulmuştur. Türlerin Küçük Menderes Nehir Havzası'nda yer alan istasyonlardaki durumu ve Türkiye florasındaki durumu karşılaştırılmıştır.

Toplamda 94 bentik diyatome türünün teşhis edildiği çalışmada kirli suların indikatörü olan Nitzschia palea (Kützing) W.Smith, N. inconspicua Grunow, N. umbonata (Ehrenberg) Lange-Bertalot, Craticula accomoda (Hustedt) DG Mann, C. subminuscula (Manguin) C.E.Wetzel \& L.Ector ve Navicula veneta Kützing en yaygın türler olmuştur. Türkiye diyatome florasında yaygın olarak bulunan Achnanthidium minutissimum, Cocconeis pediculus, Craticula accomoda, C. subminuscula, Cyclotella meneghiniana, Encyonema ventricosum, Gomphonema parvulum, Halamphora veneta, Navicula capitatoradiata, $N$. cryptocephala, $N$. gregaria, $N$. veneta, Nitzschia capitellata, $N$. inconspicua, $N$. palea, N. umbonata, Reimeria sinuata, Sellaphora pupula, S. saugerresii, Surirella brebissonii, S. robusta türlerinin Küçük Menderes Havzası'nda yaygın olarak teşhis edilmesi sebebiyle Türkiye diyatome florası ile benzerlik göstermiştir.

Diğer yandan, Craticula melostiformis, Cyclostephanos dubius, Encyonopsis minuta, E. subminuta, Gomphonema pumilum, Halamphora montana ve Mayameae permitis türleri Türkiye'de nadir olarak bulunan türler olurken bu çalışmada yaygın olarak teşhis edilmiştir. Achnanthidium eutrophilum (Lange-Bertalot) Lange-Bertalot, A. minutissimum var. jackii (Rabenhorst) LangeBertalot, Navicula simulata Manguin, N. vandamii Schoeman \& Archibald, Nitzschia archibaldii Lange-Bertalot, $N$. desertorum Hustedt ve Sellaphora saprotolerans Lange-Bertalot, Hofmann \& Cantonati türleri ise Türkiye diyatome florası için 7 yeni kayıt olmuştur. Bu türler arasında yer alan Achnanthidium eutrophilum ve Nitzschia archibaldii türleri Merkez Avrupa'da ve Almanya'da yaygın olarak görülürken Navicula vandamii türüne Almanya'da bulunan durgun sularda sık rastlanmaktadır.

Türkiye'de nehir havzalarında yürütülen biyolojik izleme faaliyetleri, su kalitesinin ekosistem esaslı olarak değerlendirilmesi açısından önemli çalışmalardır. Bu çalışmalar diğer bir yandan Türkiye flora ve faunası çalışmalarına da büyük katkı sağlamaktadır. Bugüne kadar izleme yapılmamış birçok su kütlesinde biyolojik, fizikokimyasal ve kimyasal izleme eş zamanlı olarak yapılmakta ve türlerin dağılımı ile türlerin çevresel istekleri hakkında bilgi edinilmektedir. Biyolojik izleme çalışmaları neticesinde teşhis edilen türlere ait ölçüm bilgilerinin ve havza bazında görsel katalogların oluşturulması izleme çalışmalarına büyük katkı sağlayacaktır. Bu sayede havza bazında yürütülen teşhis çalışmaları biraz daha kolaylaşacak ve böylece su kalitesinin ekolojik durumu daha hızlı bir şekilde değerlendirilebilecektir. 